\title{
Compositionality of arm movements can be realized by propagating synchrony
}

\author{
Alexander Hanuschkin • J. Michael Herrmann • \\ Abigail Morrison • Markus Diesmann
}

Received: 20 May 2010 / Revised: 2 September 2010 / Accepted: 30 September 2010 / Published online: 16 October 2010

(C) The Author(s) 2010. This article is published with open access at Springerlink.com

\begin{abstract}
We present a biologically plausible spiking neuronal network model of free monkey scribbling that reproduces experimental findings on cortical activity and the properties of the scribbling trajectory. The model is based on the idea that synfire chains can encode movement primitives. Here, we map the propagation of activity in a chain to a linearly evolving preferred velocity, which results in parabolic segments that fulfill
\end{abstract}

\section{Action Editor: David Golomb}

A. Hanuschkin · A. Morrison

Functional Neural Circuits Group, Faculty of Biology,

Schänzlestraße 1, 79104 Freiburg, Germany

\begin{abstract}
A. Hanuschkin $(\bowtie) \cdot$ A. Morrison · M. Diesmann Bernstein Center Freiburg, Freiburg, Germany

e-mail: hanuschkin@bccn.uni-freiburg.de
\end{abstract}

J. M. Herrmann

IPAB, School of Informatics,

University of Edinburgh, Edinburgh, UK

J. M. Herrmann

BCCN Göttingen and MPI for Dynamics

and Self-Organization, Göttingen, Germany

A. Morrison · M. Diesmann

Laboratory of Computational Neurophysics,

RIKEN Brain Science Institute,

Wako City, Saitama, Japan

M. Diesmann

RIKEN Computational Science Research Program,

Wako City, Saitama, Japan the two-thirds power law. Connections between chains that match the final velocity of one encoded primitive to the initial velocity of the next allow the composition of random sequences of primitives with smooth transitions. The model provides an explanation for the segmentation of the trajectory and the experimentally observed deviations of the trajectory from the parabolic shape at primitive transition sites. Furthermore, the model predicts low frequency oscillations $(<10 \mathrm{~Hz})$ of the motor cortex local field potential during ongoing movements and increasing firing rates of non-specific motor cortex neurons before movement onset.

Keywords Motor cortex • Compositionality - Synfire chains $\cdot$ LFP $\cdot$ Spike synchrony $\cdot$ Motor control

\section{Introduction}

Motor learning can be tedious. The optimisation of the interconnected processes involves the adaptation of internal models, the prediction of external rewards and the exploration of synergistic patterns of muscle activations. Often it occurs on time scales comparable to the life span and can be achieved only on the cost of the plasticity of the available motor actions. In order to reconcile the complexities of behavioural learning with the necessary flexibility, smoothness and efficiency of the movements, evolution has brought about a compositional approach that allows behavior to be generated from a set of possibly highly optimized elements. All movements that are possible for the organism can brought about by a combination of elementary movements, 
or primitives, however movement efficiency is improved by concatenating well-matched primitives in a purposeful manner.

The existence of behavioural primitives is supported by a number of experimental studies. For example, force field primitives that can be combined vectorially have been identified by stimulating the frog's spinal cord (Bizzi et al. 1991, 2008; Mussa-Ivaldi et al. 1994; Mussa-Ivaldi and Bizzi 2000). Hart and Giszter (2010) revealed the neuronal basis of these motor primitives in the spinal cord. Recently, movement primitives have been identified in a free monkey scribbling task on a two dimensional plane (Polyakov et al. 2009a, b). Segments of constant acceleration constitute the parabolic movement primitives which satisfy the two-thirds power law of the end-effector dynamics observed in experiments (Lacquaniti et al. 1983; Viviani and Flash 1995).

An obvious question arising within this approach is how primitives are represented in a dynamic network of spiking neurons. One hypothesis is based on feedforward sub-networks known as synfire chains (Abeles 1991). These structures were originally postulated to explain the occurrence of precise spike timing in cortical neurons (e.g. Eckhorn et al. 1988; Abeles et al. 1993; Prut et al. 1998). The general concept of feed-forward networks has recently been reviewed by Kumar et al. (2010) with respect to the transmission of information. Although there is substantial indirect evidence for synfire chains, a direct proof of their existence is has yet to be established due to the difficulties of determining such fine grained connectivity in neural tissue. However, methods to detect their activity continue to improve (Abeles and Gat 2001; Schrader et al. 2008; Berger et al. 2010) and theoretical and computational studies have shown that such structures would support the stable propagation of activity under quite general conditions (Herrmann et al. 1995; Diesmann et al. 1999; Gewaltig et al. 2001). Bienenstock (1995) proposed that each primitive is represented by an individual synfire chain and that the synchronization of activity between different chains binds the associated primitives into a composed object. A further study (Bienenstock 1996) elaborated on the relevance of the concept of compositionality for brain processing. Since then, it has been shown that synfire chains can be synchronized (Arnoldi and Brauer 1996) and that this mechanism can indeed realize compositionality (Hayon et al. 2005; Abeles et al. 2004; Schrader et al. 2010).

In the present study, we focus on compositionality in the sense of how behavioral primitives can be concatenated into sequences. It has been shown that networks of synfire chains can generate sequences in the context of abstract primitives (Schrader et al. 2010) or syllables in bird song ( $\mathrm{Li}$ and Greenside 2006; Jin et al. 2007; Glaze and Troyer 2008; Jin 2009; Hanuschkin et al. 2010b). We present a functional model that is simultaneously capable of reproducing several experimental findings on cortical activity and generating trajectories which exhibit key features of free monkey scribbling. Our model consists of a topologically organized network of synfire chains. Neurons in the same pool of a chain encode the same preferred velocity vector, thus realizing a population coding for movement (Georgopoulos et al. 1982, 1986b). The neural activity is characterized by asynchronous and irregular dynamics (Burns and Webb 1976; Softky and Koch 1993; van Vreeswijk and Sompolinsky 1996; Ponce-Alvarez et al. 2010), but due to the synfire activity, precise interspike timing and patterns can also be observed during ongoing motion and locked to relevant task features, as have been found experimentally in the motor cortex (Riehle et al. 1997, 2000; Shmiel et al. 2005, 2006; Ghosh et al. 2009; Putrino et al. 2010). The topological arrangement of our network is supported by the finding that correlation strength between neurons decreases as the distance between them increases (Murthy and Fetz 1996; Dombeck et al. 2009), and that nearby neurons tend to prefer similar motion parameters (Georgopoulos et al. 2007; Stark et al. 2009). The trajectories generated by our model consist of a series of parabolic segments similar to those identified experimentally (Polyakov et al. 2009a, b) which fulfill the well established two-thirds power law relationship of velocity and curvature (Lacquaniti et al. 1983; Viviani and Flash 1995). It has previously been shown that population vectors of neural activity also fulfill this relationship (Schwartz 1994).

The paper is organized as follows. In Section 2, we summarize how stable activity propagation in a feedforward network of spiking neurons can be mapped to parabolic movement primitives in position space. In Section 3, we present our model architecture and determine optimal parameters to enable long sequences of primitives. We then derive experimental predictions for the signature of synfire chain based composition of trajectories in collective signals of neural activity such as the local field potential (LFP). We analyze the characteristics of an example model trajectory and demonstrate that it shares key properties with trajectories generated by monkeys in a scribbling task. Finally, in Section 4 we discuss the model findings, limitations and predictions.

Preliminary results of this model study on free monkey scribbling have been published in abstract form (Hanuschkin et al. 2009a, b). 


\section{Materials and methods}

\subsection{Mapping uniform motion to parabolic motion}

It has previously been demonstrated that monkey scribbling is well approximated by parabolic strokes (Polyakov et al. 2009a, b). Parabolic movement primitives obey the two thirds power law, are invariant under equi-affine transformations and minimize hand jerk. A parabola can be constructed from a constant acceleration produced by a homogeneous force field. Assume the initial position and velocity of a point mass is $\mathbf{x}_{0}=\left(x_{0}, y_{0}\right)^{\mathrm{T}}$ and $\mathbf{v}_{0}=\dot{\mathbf{x}}_{0}=\left(\dot{x}_{0}, \dot{y}_{0}\right)^{\mathrm{T}}$, respectively. If the point mass experiences a constant acceleration $\mathbf{a}=\left(a_{x}, a_{y}\right)^{\mathrm{T}}$ then the trajectory of $\mathbf{x}=(x, y)^{\mathrm{T}}$ is given by

$\mathbf{x}(t)=\mathbf{x}_{0}+\mathbf{v}_{0} t+\frac{1}{2} \mathbf{a} t^{2}$.

The curvature of the path is

$c=\frac{|\dot{x} \ddot{y}-\dot{y} \ddot{x}|}{v^{3}}=\frac{\left|\dot{x}_{0} a_{y}-\dot{y}_{0} a_{x}\right|}{v^{3}}$,

where $v=\|\dot{\mathbf{x}}\|$ is the tangential velocity. Since $\mid \dot{x}_{0} a_{y}-$ $\dot{y}_{0} a_{x} \mid$ is constant, $c$ and $v$ obey the following relation

$v=K c^{-\frac{1}{3}}$

where $K$ is named velocity gain factor. Equation (2) can be rewritten in terms of the angular velocity $A=v c$ as:

$A=K c^{\frac{2}{3}}$,

the experimentally observed two-thirds power law (Lacquaniti et al. 1983). Thus, parabolic movement segments naturally fulfill the two-thirds power law.

Constant accelerations are equivalent to velocities that change linearly in time, as $\mathbf{a}=\frac{d \mathbf{v}}{d t}$. In velocity space, linearly evolving velocities $(\dot{\mathbf{v}}=$ const $)$ are represented by a uniform motion along a straight line. Therefore, straight lines in velocity space can be mapped into parabolic trajectories in position space, as illustrated in Fig. 1.

\subsection{Propagation of activity in cell assemblies}

A natural candidate neural architecture to produce spiking activity which can be associated with a uniform motion is the synfire chain (Abeles 1991). In (a)

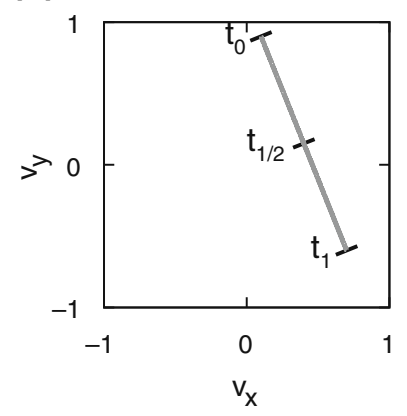

(b)

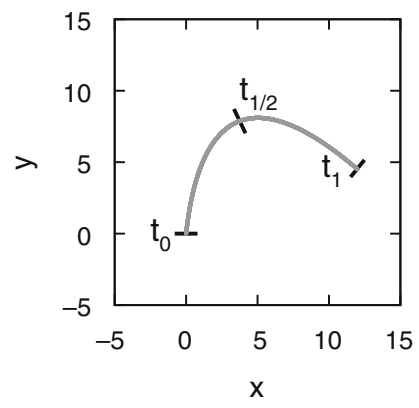

Fig. 1 Mapping uniform motion to parabolic motion. (a) Uniform motion along a straight line in velocity space corresponding to motion with constant acceleration (arbitrary units). Motion starts at time $t_{0}$ and finishes at time $t_{1}, t_{1 / 2}$ marks the middle point. (b) Parabolic trajectory in position space corresponding to (a), given an initial position $(0,0)$

the simplest formulation of the synfire chain concept, excitatory neurons are grouped in pools and each neuron is connected to all neurons of the following pool creating a chain of convergent and divergent feedforward connections. If the first group is stimulated with sufficient strength and a sufficiently high degree of synchrony, a wave of synchronous activity propagates along the chain. The propagation along the chain is at constant speed (Wennekers and Palm 1996; Diesmann et al. 1999) and stable under fairly general conditions (Herrmann et al. 1995; Diesmann et al. 1999). As

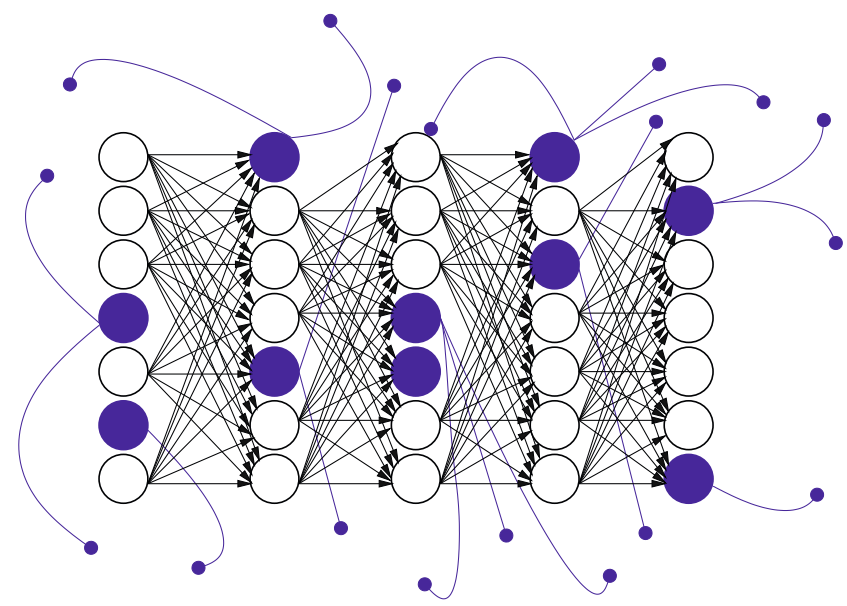

Fig. 2 Synfire chain connectivity. Excitatory neurons (open circles) of pool $i$ are connected to pool $i+1$ in a feed-forward manner. Inhibitory neurons (blue filled circles) are projecting globally to the network. Connections are visualized by arrows in the corresponding (black $=$ excitatory, blue $=$ inhibitory) colors with pointed (excitatory) or round (inhibitory) arrowheads. In this figure the dilution rate is set to $p=1$ for clarity 
illustrated in Fig. 2, the basic concept of synfire chains has recently been extended by introducing a dilution rate $p$ and globally projecting inhibitory neurons (Abeles et al. 2004; Hayon et al. 2005). Instead of connecting each neuron to all neurons of the following group, connections are drawn with probability $p$. Additionally, each synfire group comprises not only excitatory neurons making feed-forward connections, but also inhibitory neurons that make random connections to neurons selected from the entire network. The former adaptation has the effect that the propagation of activity can be more easily influenced by outside activity, such as synfire activity binding (Abeles et al. 2004; Hayon et al. 2005). The latter adaptation balances the network and enables control of the global network activity in the presence of synfire activity. The synfire chain connectivity is described in detail in Table 1, the corresponding parameters can be found in Table 3.

\subsection{Mapping activity in cell assemblies} to parabolic motion

The activity of single cells in the motor cortex has been shown to be directionally tuned to arm movements (Georgopoulos et al. 1982). The arm trajectory can be estimated by calculating the population average over all neurons (Georgopoulos et al. 1986a, 1988). Similarly,
Table 1 Summary of model structure after Nordlie et al. (2009)

\begin{tabular}{|c|c|c|c|}
\hline \multicolumn{4}{|c|}{ Model summary } \\
\hline \multicolumn{2}{|c|}{ Populations } & \multicolumn{2}{|c|}{$\begin{array}{l}\text { One network of interconnected synfire chains (SFCN), one } \\
\text { backward-and-forward connected chain (BFCN) }\end{array}$} \\
\hline \multicolumn{2}{|c|}{ Connectivity } & \multicolumn{2}{|c|}{$\begin{array}{l}\text { SFCN: excitatory feed-forward (FF) connections within each chain } \\
\text { and between final and initial groups of selected pairs of chains, } \\
\text { cross-inhibition between chains, global random inhibition. } \\
\text { BFCN: FF and feed-back (FB) connections. SFCN to BFCN: random } \\
\text { inhibition. BFCN to SFCN: FF connections from final group of BFCN } \\
\text { to initial group of chain } 1 \text { in SFCN. All connections realized } \\
\text { using random divergent (RD) or random convergent (RC) wiring. }\end{array}$} \\
\hline \multicolumn{2}{|c|}{ Neuron model } & \multicolumn{2}{|c|}{$\begin{array}{l}\text { Leaky integrate-and-fire (IaF), fixed voltage threshold, fixed absolute } \\
\text { refractory time }\end{array}$} \\
\hline \multicolumn{2}{|c|}{ Synapse model } & \multicolumn{2}{|c|}{$\alpha$-current inputs } \\
\hline \multicolumn{2}{|c|}{ Input } & \multicolumn{2}{|c|}{ Independent fixed-rate Poisson spike trains to all neurons } \\
\hline \multicolumn{2}{|c|}{ Measurements } & \multicolumn{2}{|c|}{ Spike activity, membrane potential } \\
\hline \multicolumn{4}{|c|}{ Populations } \\
\hline \multicolumn{2}{|l|}{ Name } & Elements & Size \\
\hline \multicolumn{2}{|l|}{$\overline{\mathrm{SFCN}}$} & Synfire chain $\mathrm{SFC}_{j}$ & 10 \\
\hline \multicolumn{2}{|l|}{$\mathrm{BFCN}$} & $\begin{array}{r}\text { Backward-and-forward } \\
\text { connected chain BFC }\end{array}$ & 1 \\
\hline \multicolumn{2}{|l|}{$\mathrm{SFC}_{j}$} & SFC groups $G_{i, j}$ & 50 \\
\hline \multicolumn{2}{|l|}{$\mathrm{BFC}$} & BFC groups $G_{k}^{*}$ & 50 \\
\hline \multicolumn{2}{|l|}{$G_{i, j}$} & Neuron populations $\mathrm{E}_{i, j}, \mathrm{I}_{i, j}$ & 2 populations per group $G_{i, j}: \mathrm{E}_{i, j}$ and $\mathrm{I}_{i, j}$ \\
\hline \multicolumn{2}{|l|}{$G_{k}^{*}$} & Neuron populations $\mathrm{E}_{k}^{*}, \mathrm{I}_{k}^{*}$ & 2 population per group $G_{k}^{*}: \mathrm{E}_{k}^{*}$ and $\mathrm{I}_{k}^{*}$ \\
\hline \multicolumn{2}{|l|}{$\mathrm{E}_{i, j}^{\kappa}, \mathrm{E}_{k}^{*}$} & Excitatory IaF neuron & 100 \\
\hline \multicolumn{2}{|l|}{$\mathrm{I}_{i, j}, \mathrm{I}_{k}^{*}$} & Inhibitory IaF neuron & 25 \\
\hline \multicolumn{4}{|c|}{ Connectivity } \\
\hline Name & Source & Target & Pattern \\
\hline$\overline{F F}$ & $\mathrm{E}_{i, j}$ & $\mathrm{E}_{i+1, j}+\mathrm{I}_{i+1, j}$ & $\mathrm{RD}, 1 \rightarrow C_{\mathrm{Ex}}$, weight $J_{\mathrm{E}}$, delay $d$ \\
\hline$C h_{\text {conn }}$ & $\mathrm{E}_{50, j}$ & $\mathrm{E}_{1, j^{\prime}}+\mathrm{I}_{1, j^{\prime}}$, with $j, j^{\prime}$ as Fig. 4 & $\mathrm{RC}, C_{\mathrm{Ex}} \rightarrow 1$, weight $J_{\mathrm{E}}$, delay $d$ \\
\hline$I_{\text {global }}$ & $\mathrm{I}_{i, j}$ & $\mathrm{SFC}_{j}$ & $\mathrm{RD}, 1 \rightarrow k_{\mathrm{g}}$, weight $J_{\mathrm{I}}$, delay $d$ \\
\hline$I_{\text {cross }}$ & $\mathrm{I}_{i, j}$ & $\mathrm{SFC}_{j^{\prime}}$, with $j, j^{\prime}$ as Fig. 4 & $\mathrm{RD}, 1 \rightarrow k_{\mathrm{c}}$, weight $J_{\mathrm{I}}$, delay $d$ \\
\hline$I_{\mathrm{BFC}}$ & $\mathrm{I}_{i, j}$ & $\mathrm{BFC}$ & $\mathrm{RD}, 1 \rightarrow k_{\mathrm{B}}$, weight $J_{\mathrm{I}}$, delay $d$ \\
\hline$F F^{*}$ & $\mathrm{E}_{k}^{*}$ & $\mathrm{E}_{k+1}^{*}+\mathrm{I}_{k+1}^{*}$ & $\mathrm{RD}, 1 \rightarrow C_{\mathrm{Ex}}$, weight $J_{\mathrm{E}}$, delay $d$ \\
\hline$F B^{*}$ & $\mathrm{E}_{k}^{*}$ & $\mathrm{E}_{k-1}^{*}+\mathrm{I}_{k-1}^{*}$ & $\mathrm{RD}, 1 \rightarrow C_{\mathrm{Ex}}$, weight $J_{\mathrm{E}}$, delay $d$ \\
\hline$I_{\text {global }}^{*}$ & $\mathrm{I}_{k}^{*}$ & $\mathrm{BFC}$ & $\mathrm{RD}, 1 \rightarrow k_{\mathrm{g}}^{*}$, weight $J_{\mathrm{I}}$, delay $d$ \\
\hline$E_{\mathrm{SFC}}^{*}$ & $\mathrm{E}_{50}^{*}$ & $\mathrm{SFC}_{1,1}$ & $\mathrm{RC}, C_{\mathrm{Ex}} \rightarrow 1$, weight $J_{\mathrm{E}}$, delay $d$ \\
\hline
\end{tabular}


we use population coding to generate a trajectory from simulated neuronal activity:

$$
\begin{aligned}
\mathbf{v} & =\sum_{k}^{\text {all neurons }} w_{k} a_{k}(t) \mathbf{p}_{k} \\
& =\sum_{j}^{\text {chain pool }} \sum_{i} w_{i}^{j} a_{i}^{j}(t) \mathbf{p}_{i}^{j},
\end{aligned}
$$

where $\mathbf{v}$ is the instantaneous velocity, $a_{i}^{j}(t)$ is the activity in the $i$ th group of the $j$ th chain and $\mathbf{p}_{i}^{j}$ its preferred velocity. The weights are set to $w_{i}^{j}=0.02 \mathrm{~s} \forall i, j$ resulting in velocities comparable to the monkey experiments (median $300 \mathrm{~mm} / \mathrm{s}$ as given by Polyakov et al. 2009b). The propagation speed of the activity volley in a synfire chain from one pool to the next is constant as described in Section 2.2. We can therefore map a synfire chain to an arrow in velocity space. Each pool of the synfire chain is assigned its preferred velocity $\mathbf{p}_{i}$ according to its position along the arrow, i.e. for a chain consisting of $n$ pools mapped to an arrow starting at $\mathbf{v}_{0}$ and ending at $\mathbf{v}_{1}$,

$\mathbf{p}_{i}=\frac{i-1}{n-1}\left(\mathbf{v}_{1}-\mathbf{v}_{0}\right)+\mathbf{v}_{0}$.

This is illustrated in Fig. 3(a); the activity of the corresponding synfire chain is given in Fig. 3(b). As the preferred velocity for each chain $j$ changes linearly with the pool index $i$ and the propagation speed from one pool to the next is constant, the instantaneous velocity vector also evolves linearly resulting in parabolic motion as derived in Section 2.1. Figure 3(c) shows the parabolic trajectory in position space generated by the synfire activity in Fig. 3(b). To extract the trajectory from the simulated neuronal activity, we bin the activity in $1 \mathrm{~ms}$ intervals and reconstruct the motion according to the population coding scheme given by Eqs. 4 and 5 .

\subsection{Sequences of primitives}

As discussed in the previous section, an appropriate mapping of preferred velocities to the pools of a synfire chain enables the generation of an individual parabolic segment. By extension, a series of parabolic strokes in position space can be realized by uniform motion along a graph of connected straight lines in velocity space. An example of this is shown in Fig. 4. Each arrow in velocity space is realized by a synfire chain with the corresponding velocity mapping. By construction, the velocity at the end of one parabolic stroke is equal to the velocity at the beginning of the next. When the activity volley in a synfire chain reaches the final pool, feed-forward connections to the initial pools of the two potential successor chains initiates the propagation of an activity volley in each of them. Assuming a strong competition between the two stimulated chains, such that only one of the chains can continue
Fig. 3 Mapping synfire activity to parabolic movements: (a) The preferred velocity vectors for the pools of the synfire chain (gray arrows; shown for every third pool of the chain) are determined by sampling a straight line in velocity space (red arrow). (b) The spiking activity of an activity volley propagating with constant speed along a synfire chain. Preferred velocity vectors for every third pool as in (a) are shown as gray arrows above the dot display. (c) Generated parabolic trajectory. The black cross at $(0,0)$ indicates the start position
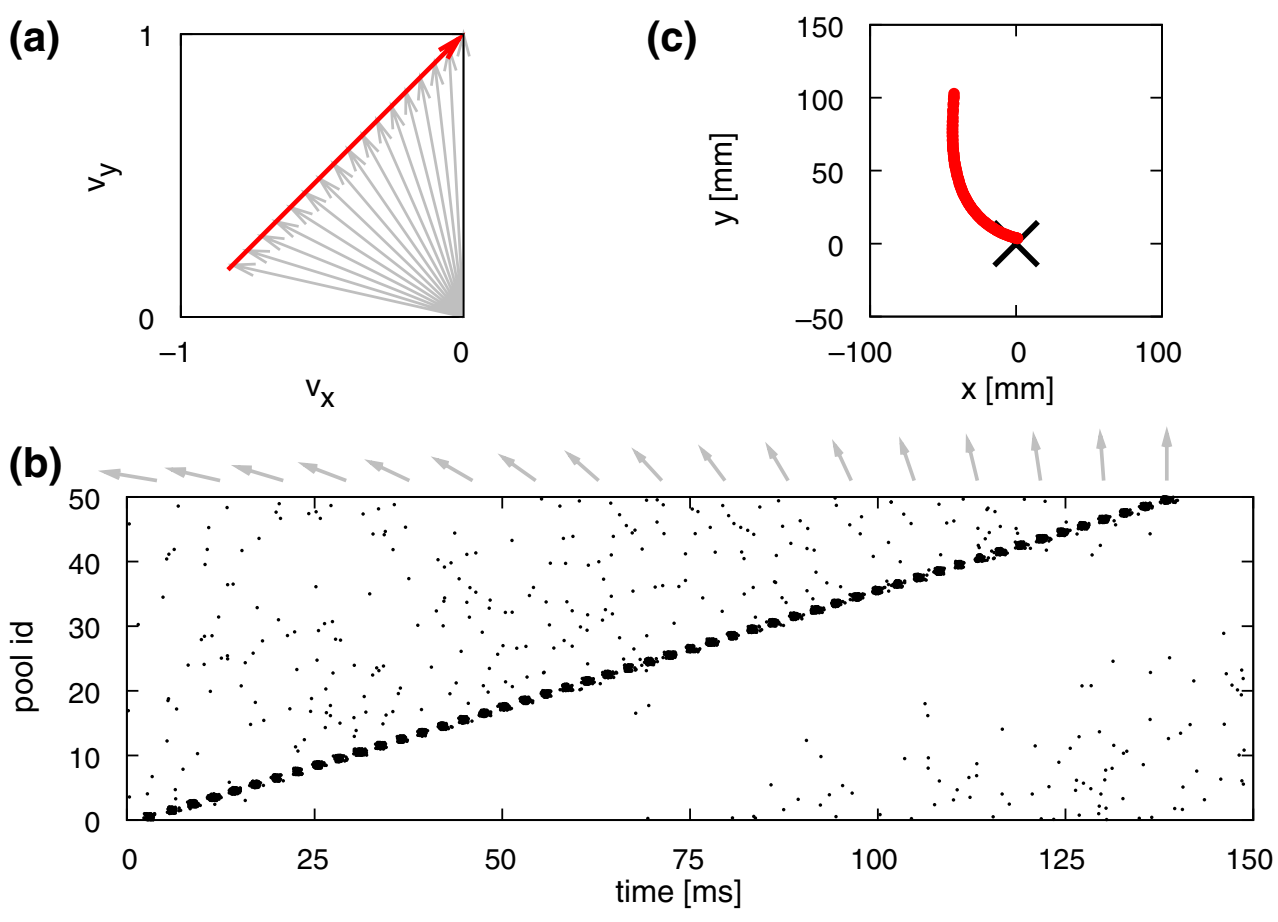


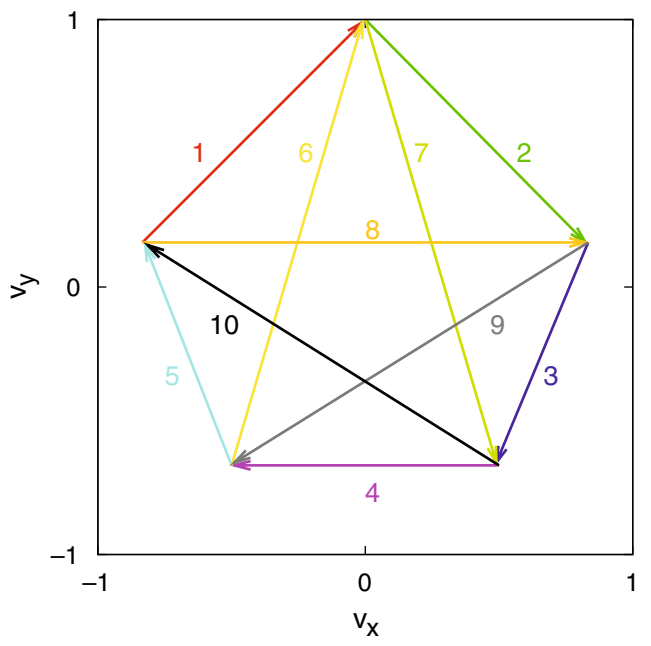

Fig. 4 Abstract generator for trajectories consisting of parabolic segments. Uniform motion along straight lines in velocity space (arbitrary units) is equivalent to parabolic motion in position space. Each colored arrow represents a parabolic segment and its direction of execution. When the end of an arrow is reached, one of the two successor arrows is selected. Arrows are numbered to enable future reference

propagating the activity, a trajectory of parabolic segments is produced.

\subsection{Analysis of the trajectories}

We analyze the properties of the generated trajectories to see whether they are sequences of parabolic segments. A parabola has zero equi-affine curvature (for a brief introduction to equi-affine geometry see Polyakov et al. 2009b). Following Calabi et al. (1996, 1998), the equi-affine curvature $\kappa$ can be approximated employing five points $P_{n}$. We define

$$
[i j k]=\left[P_{i}, P_{j}, P_{k}\right]=\operatorname{det}\left|\begin{array}{lll}
x_{i} & y_{i} & 1 \\
x_{j} & y_{j} & 1 \\
x_{k} & y_{k} & 1
\end{array}\right|
$$

which is twice the signed area of the triangle with vertices $P_{i}, P_{j}$ and $P_{k}$. The equi-affine curvature $\kappa$ is now given by:

$\kappa=\frac{S}{T^{\frac{2}{3}}}$,

where

$$
4 T=\prod_{0 \leq i<j<k \leq 4}[i j k]
$$

and

$$
\begin{aligned}
4 S= & {[013]^{2}[024]^{2}([124]-[123])^{2} } \\
& +[012]^{2}[034]^{2}([134]-[132])^{2} \\
& -2[012][034][013][024] \cdot([123][234] \\
& +[124][134]) .
\end{aligned}
$$

\subsection{Numerical simulations}

We perform numerical simulations of networks of passive leaky integrate-and-fire neurons (Lapicque 1907; Abbott 1999) with post synaptic currents (PSCs) described by the alpha-function. This model provides a

\begin{tabular}{|c|c|}
\hline \multicolumn{2}{|l|}{ Neuron models } \\
\hline Name & IaF neuron \\
\hline Type & Leaky integrate-and-fire, $\alpha$-current input \\
\hline Subthreshold dynamics & $\begin{aligned} \tau_{m} \dot{V}(t) & =-V(t)+R I(t) \quad \text { if } t>t^{*}+\tau_{\mathrm{ref}} \\
V(t) & =V_{0} \quad \text { else } \\
I(t) & =I_{0}+\hat{i} \frac{e}{\tau_{\alpha}} \sum_{\tilde{t}} e^{\frac{t-\tilde{t}}{\tau_{\alpha}}} \delta(t-\tilde{t})\end{aligned}$ \\
\hline \multirow[t]{2}{*}{ Spiking } & $\begin{array}{l}\text { If } V(t-)<V_{\text {th }} \wedge V(t+) \geq V_{\text {th }} \\
\text { 1. calculate retrospective threshold crossing } \\
\text { with bisectioning method (Hanuschkin et al. 2010c) }\end{array}$ \\
\hline & $\begin{array}{l}\text { 2. set } t^{*}=t+\Delta_{\text {offset }} \\
\text { 3. emit spike with time stamp } t^{*}\end{array}$ \\
\hline \multicolumn{2}{|l|}{ Input } \\
\hline Type & Description \\
\hline Poisson generator & Independent for all targets, rate $v_{\mathrm{x}}$, weight $J_{\mathrm{E}}$ \\
\hline \multicolumn{2}{|c|}{ Measurements } \\
\hline \multicolumn{2}{|c|}{ Spike activity of all neurons, membrane potential of neurons of $\operatorname{SFC}_{j}$ with $j \in(1,2,7)$} \\
\hline
\end{tabular}
reasonable approximation of many of the basic features of cortical nerve cells, such as integration of
Table 2 Summary of model dynamics after Nordlie et al. (2009) 
synaptic inputs, and the stereotypical character of the action potential and can be simulated efficiently (Rotter and Diesmann 1999; Plesser and Diesmann 2009) since the sub threshold membrane dynamic is expressed by a time invariant linear differential equation. This enables us to simulate networks of ten thousands of neurons with biological realistic connectivity necessary for the investigation of collective phenomenas like the propagation of ensemble firing of neuronal groups. A description of the neuron model dynamics and corresponding parameters can be found in Tables 2 and 3 .

Simulations were performed with NEST revision 8257 (see www.nest-initiative.org and Gewaltig and Diesmann 2007) using a computational step size of $1 \mathrm{~ms}$ on a standard workstation running Linux. To avoid synchrony artefacts (Hansel et al. 1998), we employ precise simulation techniques in a globally time driven framework (Morrison et al. 2007b; Hanuschkin et al. 2010c). To allow other researchers to perform their own experiments, at the time of publication we are making a module available for download at www.nest-initiative.org containing all relevant scripts.

Table 3 Specification of model parameters

\begin{tabular}{|c|c|c|}
\hline Name & Value & Description \\
\hline \multicolumn{3}{|c|}{ Connectivity } \\
\hline$C_{\mathrm{Ex}}$ & 93 & $\begin{array}{l}\text { Number of feed-forward connections } \\
\text { from each excitatory neuron }\end{array}$ \\
\hline$k_{\mathrm{g}}$ & 7 & $\begin{array}{l}\text { Number of outgoing global connections } \\
\text { from each inhibitory neuron }\end{array}$ \\
\hline$k_{\mathrm{c}}$ & 19 & $\begin{array}{l}\text { Number of cross connections } \\
\text { from inhibitory neuron to each } \\
\text { competing } \mathrm{SFC}_{j}\end{array}$ \\
\hline$k_{\mathrm{B}}$ & 6 & $\begin{array}{l}\text { Number of connections from each } \\
\text { inhibitory neuron to the BFCN }\end{array}$ \\
\hline$k_{\mathrm{g}}^{*}$ & 0 & $\begin{array}{l}\text { Number of connections from each } \\
\text { inhibitory neuron in the BFCN }\end{array}$ \\
\hline$J_{E}$ & $20.68 \mathrm{pA}$ & $\begin{array}{l}\text { Amplitude of excitatory connection, } \\
\Rightarrow 0.1 \mathrm{mV} \text { EPSP amplitude }\end{array}$ \\
\hline$J_{I}$ & $-124.68 \mathrm{pA}$ & $\begin{array}{l}\text { Amplitude of inhibitory connection, } \\
\Rightarrow-0.6 \mathrm{mV} \text { EPSP amplitude }\end{array}$ \\
\hline$d$ & $1.5 \mathrm{~ms}$ & Synaptic transmission delay \\
\hline \multicolumn{3}{|c|}{ Neuron model } \\
\hline$\tau_{\mathrm{m}}$ & $20 \mathrm{~ms}$ & Membrane time constant \\
\hline$C_{\mathrm{m}}$ & $250 \mathrm{pF}$ & Membrane capacitance \\
\hline$V_{\text {th }}$ & $20 \mathrm{mV}$ & Fixed firing threshold \\
\hline$V_{0}$ & $0 \mathrm{mV}$ & Resting potential \\
\hline$V_{\text {reset }}$ & $0 \mathrm{mV}$ & Reset potential \\
\hline$\tau_{\text {ref }}$ & $2 \mathrm{~ms}$ & Absolute refractory period \\
\hline$\tau_{\alpha}$ & $0.5 \mathrm{~ms}$ & Rise time of post-synaptic current \\
\hline \multicolumn{3}{|l|}{ Input } \\
\hline$v_{\mathrm{x}}$ & $7.7 \mathrm{kHz}$ & External Poisson rate \\
\hline
\end{tabular}

The categories correspond to the model summary in Tables 1 and 2

\section{Results}

\subsection{Network model architecture}

We develop a spiking network model to realize a generator of random trajectories consisting of parabolic segments. Our model comprises two interconnected networks as shown in Fig. 5. The synfire chain network (SFCN) consists of ten chains, each chain corresponding to one of the arrows in velocity space shown in Fig. 4 and thus encoding a parabolic segment. Each chain consists of $80 \%$ excitatory neurons that make feed-forward connections with dilution factor $p=0.75$ and $20 \%$ inhibitory neurons making $k_{\mathrm{g}}$ random connections to other neurons in SFCN. To distinguish the random inhibitory connections from other connectivity patterns, we will refer to $k_{\mathrm{g}}$ as the global inhibition parameter. The graph vertices specified in Fig. 4 are realized by feed-forward connections from the final group of each chain to the initial groups of two other chains, e.g. the final group of chain 1 has feed-forward connections to the initial groups of chains 2 and 7 . The preferred velocity of the last group of a chain is the same as the first groups of the chains it connects to in order to generate trajectories that are smooth at the transition points. Reliable switching at the transition points is enabled by mutual inhibition between potential successor chains; this is discussed in detail in Section 3.2.

In strongly recurrently connected networks of spiking neurons synfire activity can be ignited spontaneously (Tetzlaff et al. 2002). Although this is an

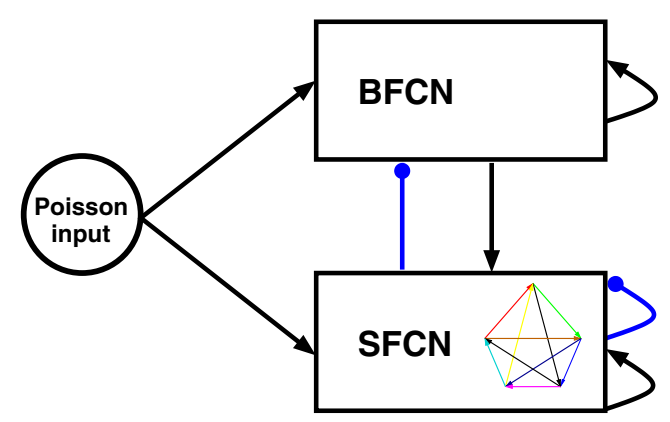

Fig. 5 Architecture of the network model. Two networks are coupled with an inhibitory/excitatory reciprocal connection. Excitatory connections are shown as black pointed arrows, inhibitory connections as blue rounded arrows. The synfire chain network (SFCN) consists of 10 synfire chains connected into a directed graph as shown in Fig. 4. The backward-and-forward connected network (BFCN) consists of a single backward-andforward connected chain. Both networks are driven by an independent excitatory Poisson input to each neuron with rate $v_{\mathrm{x}}=7.7 \mathrm{kHz}$ 
unwanted effect in studies investigating embedded synfire chains in balanced recurrent networks as it limits the density of local feed forward structures, we exploit this property in our model. We create a synfire chain with feed-backward as well as feed-forward connections, both with a dilution factor of $p$. This backward-and-forward connected chain, or BFC, constitutes the second network (BFCN). One end of the BFC makes excitatory feed-forward connections with dilution factor $p$ to the initial pool of chain 1 in SFCN. Each inhibitory neuron in SFCN makes $k_{\mathrm{B}}$ connections to neurons randomly selected from BFCN, thus inhibiting its activity when synfire activity is present. If synfire activity is extinguished, the drop in inhibition causes a self-ignition in the unstable BFC, which in turn triggers a fresh wave of activity in chain 1 . Thus the recurrent connections between SFCN and the BFCN ensure sustained activity. The dynamics of the BFCN and of the interaction with SFCN are investigated in Section 3.3. The arbitrary choice of chain 1 to re-ignite the SFCN is made here for the sake of simplicity; a more complex interaction could be assumed which would allow reignition of the SFCN at the beginning of any chain.

The scaling of inhibitory synapses with respect to excitatory synapses and the rate of the external excitatory Poisson input to each neuron in SFCN are chosen such that in the absence of synfire activity, the network spikes in the asynchronous irregular (AI) regime (Brunel 2000). A tabular description of our model is given in Tables 1 and 2; unless otherwise stated, model parameters are as given in Table 3 .

\subsection{Competition between synfire chains}

Each of the chains in SFCN represents a parabolic movement primitive. To produce a series of primitives, it is necessary that activity reliably propagates from one chain to exactly one of multiple (here two) potential successor chains at the vertices of the network graph (see Fig. 4). In our model, cross-inhibition realizes this switching between two simultaneously activated and competing chains. We investigate two approaches to achieve reliable switching. In Section 3.2.1, crossinhibition is structured such that synchronous activity in each pool directly inhibits the activity in the next pool of the competitor chain. This approach is motivated by the idea of synfire binding (Abeles et al. 2004; Hayon et al. 2005; Schrader et al. 2010), in which two simultaneously active chains can bind a third chain due to structured excitation. In an alternative approach in Section 3.2.2, the cross-inhibition is unstructured. Synfire chain competition relying solely on global inhibition has recently been proposed by Chang and Jin (2009).
However, in their study the synfire chain activity is 'driven': a suprathreshold driving input is combined with dominant global inhibition. In contrast, our model exhibits activity in the asynchronous irregular regime due to balanced global inhibition (van Vreeswijk and Sompolinsky 1996) and only exhibits synfire activity if the initial pool of a chain receives additional stimulation. Due to our different activity regime, additional assumptions on the inhibition between chains need to be made to realize reliable switching.

\subsubsection{Competition by structured cross-inhibition}

Figure 6 illustrates the structured cross-inhibition architecture. Each neuron in the initial pools of the potential successor chains is activated by $p \cdot C_{\mathrm{E}}$ randomly chosen excitatory neurons from the final pool of the preceding chain. The symmetric connections ensure that the successor chains are stimulated equally. All inhibitory neurons of pool $i$ of one potential successor chain project to $k_{\mathrm{c}}$ neurons of pool $i+1$ of the other potential successor chain, and vice versa. Thus each wave of synchronous activity directly inhibits the propagation of the activity to the next pool in the competitor chain, leading to a competition. The activity in the losing chain dies away leaving the activity in the winning chain to continue propagating, thus realizing a switching mechanism.

To demonstrate the switching and its effects on collective signals such as average firing rate or local field potential (LFP), we change the inter-chain connectivity such that only chains 1,2 and 7 can be activated. A reduced network which still exhibits synfire chain competition is provided by the following connectivity: the final pool of chain 1 is connected to the initial pools of chains 2 and 7, the final pools of these chains are connected back to the initial pool of chain 1. Chains 2 and 7 are mutually cross inhibited as shown in Fig. 6.

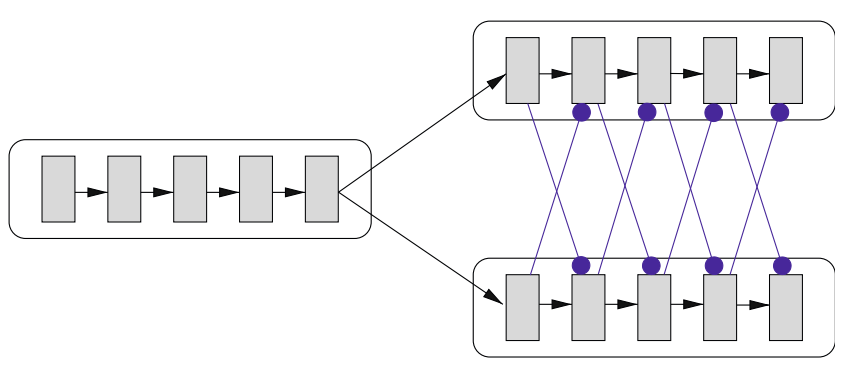

Fig. 6 Structured cross-inhibition architecture. The final pool of one synfire chain activates the initial pools of two potential successor chains (excitatory connections illustrated by black pointed arrows). Each pool in a successor chain inhibits the next pool in the competitor chain (blue rounded arrows) 
A reduced network with no synfire chain competition is realized by connecting chains 1,2 and 7 in a cyclical fashion but retaining the structured cross-inhibition between chains 2 and 7. Both reduced networks are activated by an external Gaussian pulse packet to the initial group of chain 1 . We calculate the average firing rate (Gaussian smoothing kernel with $\sigma=1 \mathrm{~ms}$ ) and two approximations of the local field potential (LFP) of the network. A first approximation of the LFP is given by the average membrane potential of $10 \%$ of the neurons in the network (Ursino and Cara 2006). A more sophisticated approximation of the LFP (in arbitrary units) is given by calculating the sum of the absolute values of the excitatory and inhibitory postsynaptic currents (PSCs) in $1 \mathrm{~ms}$ steps and smoothing the result with a Gaussian filter with $\sigma=1 \mathrm{~ms}$ (Mazzoni et al. 2008).

The switching mechanism is demonstrated in Fig. 7(a), which shows the spiking activity of the minimal network with synfire chain competition. After each completion of chain 1 , either chain 2 or chain 7 wins the competition; the activity in the other chain dies away. The average firing rate, mean potential and approximated LFP of the network are given in Fig. 7(b). The average firing rate increases whenever two chains are competing, returning to its initial value after the activity of the losing chain has died away. The time of cell assembly competition is also clearly visible in both LFP approximations. The average membrane potential shows a dip right after the increased local firing rate due to the neuron reset and refractory period during which the membrane potential is clamped to zero. The change in the signal given by the summed and smoothed absolute postsynaptic currents is particularly pronounced at the transition sites due to the contribution of the inhibitory PSCs, which are $g$ times larger than the excitatory currents. Figure 7(c) shows the spiking activity for the corresponding network without synfire chain competition. The activity moves from one chain to the next in a deterministic fashion. Figure 7(d) shows the corresponding collective signals. The approximated LFP signal still exhibits step-like changes due to the increase of inhibitory activity when either chain 2 or 7 is active, however the transient increases of LFP signal and firing rate and transient decreases of membrane potential indicated by arrows in Fig. 7(b) are not observed in the reduced network without synfire chain competition. These results suggests that low frequency components of the LFP in experimental data could be indicators for cell assembly competition.

We now determine the optimal values for $k_{\mathrm{g}}$ and $k_{\mathrm{c}}$, the number of outgoing connections that each inhibitory neuron in a synfire chain establishes with randomly selected neurons and with neurons in the competitor chain, respectively. To this end, we investigate the switching error in a network where the final pool of chain 1 is connected to the initial pools of chains 2 and 7 , and the final pools of chains 2 and 7 have no outgoing
Fig. 7 The effect of synfire chain switching realized by structured cross-inhibition on collective signals.

(a) Spiking activity of a reduced network with synfire chain competition, connected as $1 \rightarrow 2,7$ and $2,7 \rightarrow 1$. Activity of $10 \%$ of the neurons is shown. (b) Firing rate (black), average membrane potential (red) and approximated LFP (blue; arbitrary units) calculated from activity in the reduced network. Arrows indicate signatures of synfire chain switching in the collective signals. (c, d) As in $(\mathbf{a}, \mathbf{b})$ but without synfire chain competition: connected as $1 \rightarrow 2 \rightarrow 7 \rightarrow 1$. Arrows indicate regimes in which cross-inhibition is absent (1) and present (2)

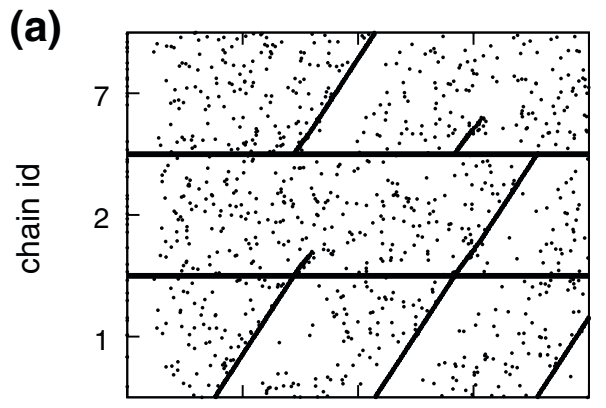

(c)

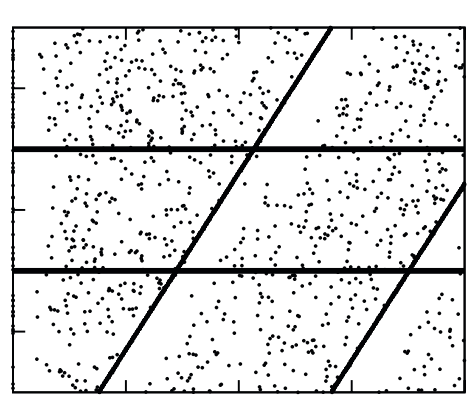

(b)

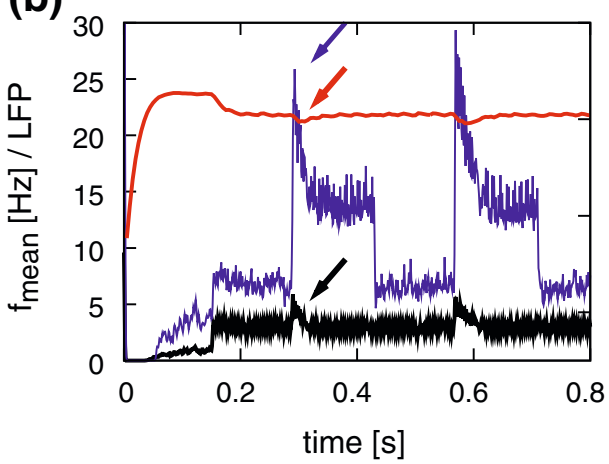

(d)

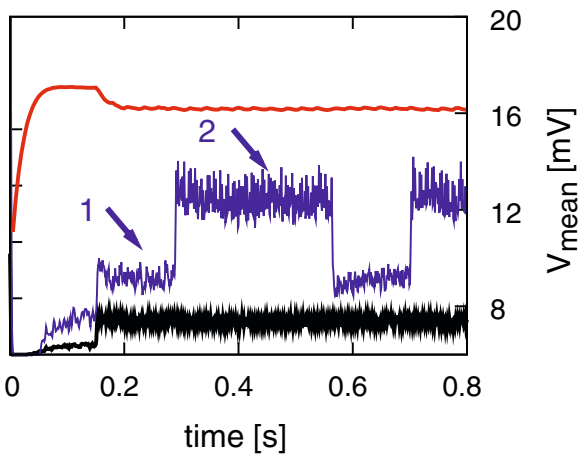


feed-forward connections. For a given configuration of $k_{\mathrm{g}}$ and $k_{\mathrm{c}}$, the network is stimulated 100 times by applying a Gaussian pulse packet to the initial pool of chain 1 . We record the occurrence of the two types of switching error: the activation of both successor chains as depicted in Fig. 8(a) and the activation of neither of the successor chains as illustrated in Fig. 8(b).

Figure $8(\mathrm{c})$ shows the probability of activating both successor chains and Fig. 8(d) illustrates the probability of neither of the successor chains as functions of $k_{\mathrm{g}}$ and $k_{\mathrm{c}}$. As the cross-inhibition parameter $k_{\mathrm{c}}$ and the global inhibition parameter $k_{\mathrm{g}}$ increase, the probability of activating both successor chains decreases whilst the probability that neither chain is activated increases. The cross-inhibition parameter $k_{\mathrm{c}}$ directly influences the competition of the chains and so has a stronger effect on the switching behavior than the global inhibition $k_{\mathrm{g}}$ parameter, which regulates the overall activity in the network. A good choice for the number of inhibitory connections is $k_{\mathrm{c}}=7$ and $k_{\mathrm{g}}=7$ : for this configuration, the probability of activating both successor chains is $p_{2}=5.44 \% \pm 2.26$, whereas the probability that neither chain is activated is $p_{0}=$
$4.98 \% \pm 1.85$. The means and standard deviations for these two values are calculated by performing the 100 switching trials for 100 different network realizations. This configuration exhibits a reasonable compromise between reliable switching and stability. The probability of activating two chains can be reduced to zero by increasing $k_{\mathrm{c}}$, but at the cost of increasing the probability of activating neither chain. Likewise, the probability of activating neither chain reaches zero for small $k_{\mathrm{c}}$, but the probability of activating both chains increases. For a given realization of the network a bias towards selecting chain 2 or chain 7 can be observed, however on average there is no bias due to the symmetry of the randomly chosen connections (data not shown).

\subsubsection{Competition by unstructured cross-inhibition}

Figure 9 illustrates the unstructured cross-inhibition architecture. As above, each neuron in the initial pools of the potential successor chains is activated by $p \cdot C_{\mathrm{E}}$ randomly chosen excitatory neurons from the final pool of the preceding chain and symmetric connections ensure that the successor chains are stimulated equally.

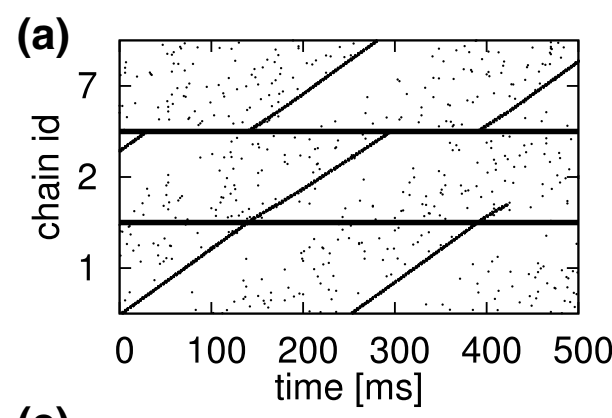

(c)

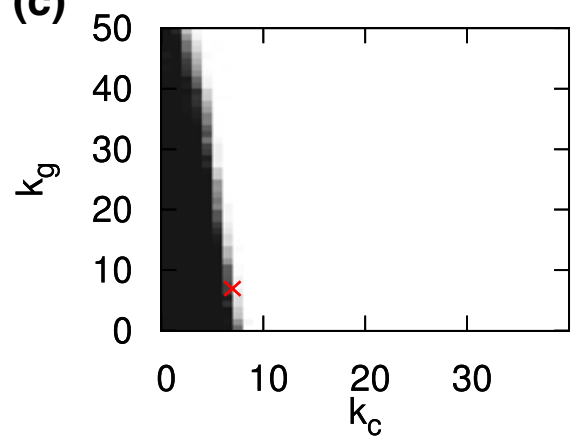

Fig. 8 Switching errors as a function of the number of inhibitory cross connections $k_{\mathrm{c}}$ and global inhibitory connections $k_{\mathrm{g}}$. (a) Spiking activity of a reduced network connected as $1 \rightarrow 2,7$ with $k_{\mathrm{c}}=k_{g}=7$. Activity of $10 \%$ of the neurons is shown. A switching error in which both successor chains are activated occurs at around $t=140 \mathrm{~ms}$. (b) As in (a), but depicting a switching error in which neither successor chain is activated at around (b)

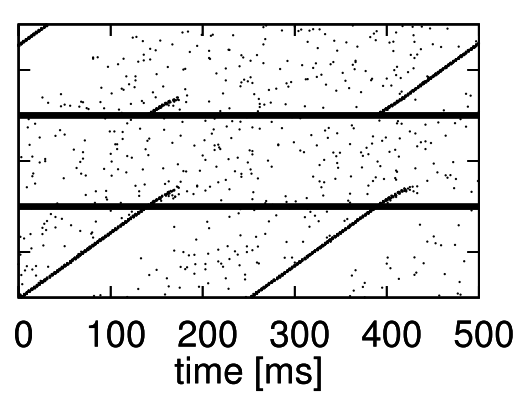

(d)

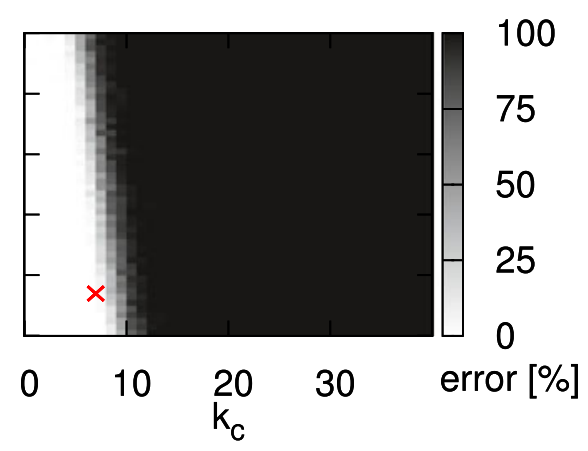

$t=140 \mathrm{~ms}$. (c) Percentage of trials resulting in the activation of both successor chains as a function of the number of inhibitory cross connections (horizontal axis) and the number of global inhibitory connections (vertical axis). (d) As in (c) but for the case that neither of the successor chains is activated. The red cross indicates the chosen parameter set $k_{\mathrm{c}}=k_{g}=7$ 


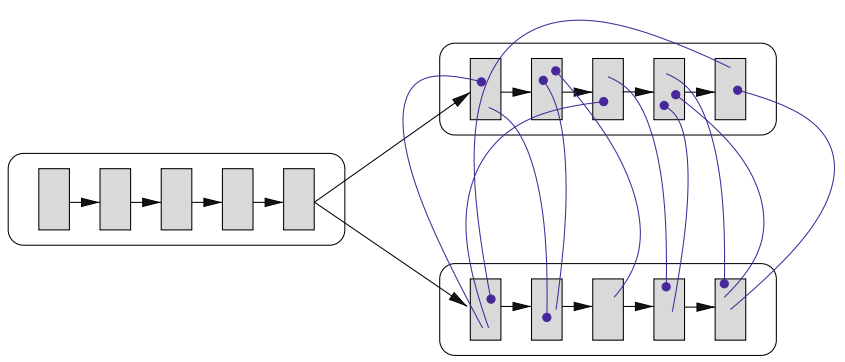

Fig. 9 Unstructured cross-inhibition architecture. The final pool of one synfire chain activates the initial pools of two potential successor chains (excitatory connections illustrated by black pointed arrows). Each inhibitory neuron in a successor chain projects to a number of randomly chosen neurons in the competitor chain (blue rounded arrows)

All inhibitory neurons of one potential successor chain project to $k_{\mathrm{c}}$ randomly chosen neurons from the other potential successor chain, and vice versa. This architecture uniformly inhibits synfire activity in the competitor, rather than simply the propagation to the next pool as in Section 3.2.1. This architecture also leads to a competition between the activated synfire chains, such that the activity in the losing chain dies away and the activity in the winning chain continues propagating.

Figure 10 demonstrates synfire chain switching on the basis of unstructured cross-inhibition and its effect on collective signals. The reduced network connectivity and the spike data analysis are as previously described in Section 3.2.1.
As in Fig. 7(b), the average firing rate in Fig. 10(b) increases whenever two chains are competing, returning to its initial value after the activity of the losing chain has died away. The time of cell assembly competition is also clearly visible in both LFP approximations as indicated by the red and blue arrows. Figure 10(c) shows the spiking activity for the corresponding network without synfire chain competition. As for the structured cross-inhibition case, Fig. 10(d) illustrates that the effects marked by arrows in Fig. 10(b) do not occur in the absence of competition between the synfire chains. In this case, a step-like modulation is visible for the average membrane potential as well as the approximated LFP. These steps are not observed during simulations of the full network model, as each chain competes with some other chain and thus all chains generate the same excitatory and inhibitory activity. However, they might be observable in a less symmetric model where chains may have differing numbers of potential successor chains.

As in the case of structured cross-inhibition, to find the optimal values for the number of outgoing connections that each inhibitory neuron in a synfire chain establishes with $k_{\mathrm{g}}$ randomly selected neurons and $k_{\mathrm{c}}$ neurons in its competitor chain, we investigate the switching error in a network where the final pool of chain 1 is connected to the initial pools of chains 2 and 7, and the final pools of chains 2 and 7 have no outgoing feedforward connections. Figure 11 shows the probabilities
Fig. 10 The effect of synfire chain switching realized by unstructured cross-inhibition on collective signals.

(a) Spiking activity of $10 \%$ of the neurons of a reduced network with synfire chain competition, connected as $1 \rightarrow 2,7$ and $2,7 \rightarrow 1$. (b) Firing rate (black), average membrane potential (red) and approximated LFP (blue; arbitrary units) calculated from activity in the reduced network. Arrows indicate signatures of synfire chain switching in the collective signals. (c, d) As in $(\mathbf{a}, \mathbf{b})$ but without synfire chain competition: connected as $1 \rightarrow 2 \rightarrow 7 \rightarrow 1$

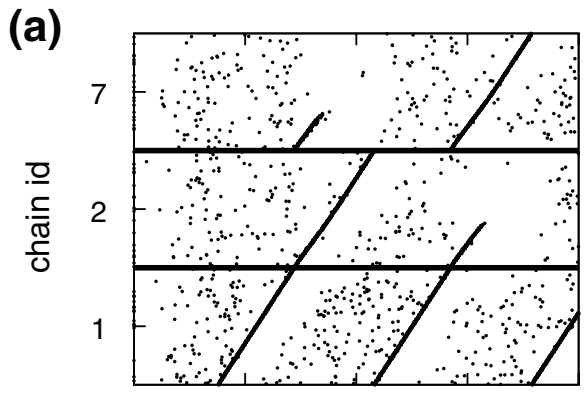

(c)
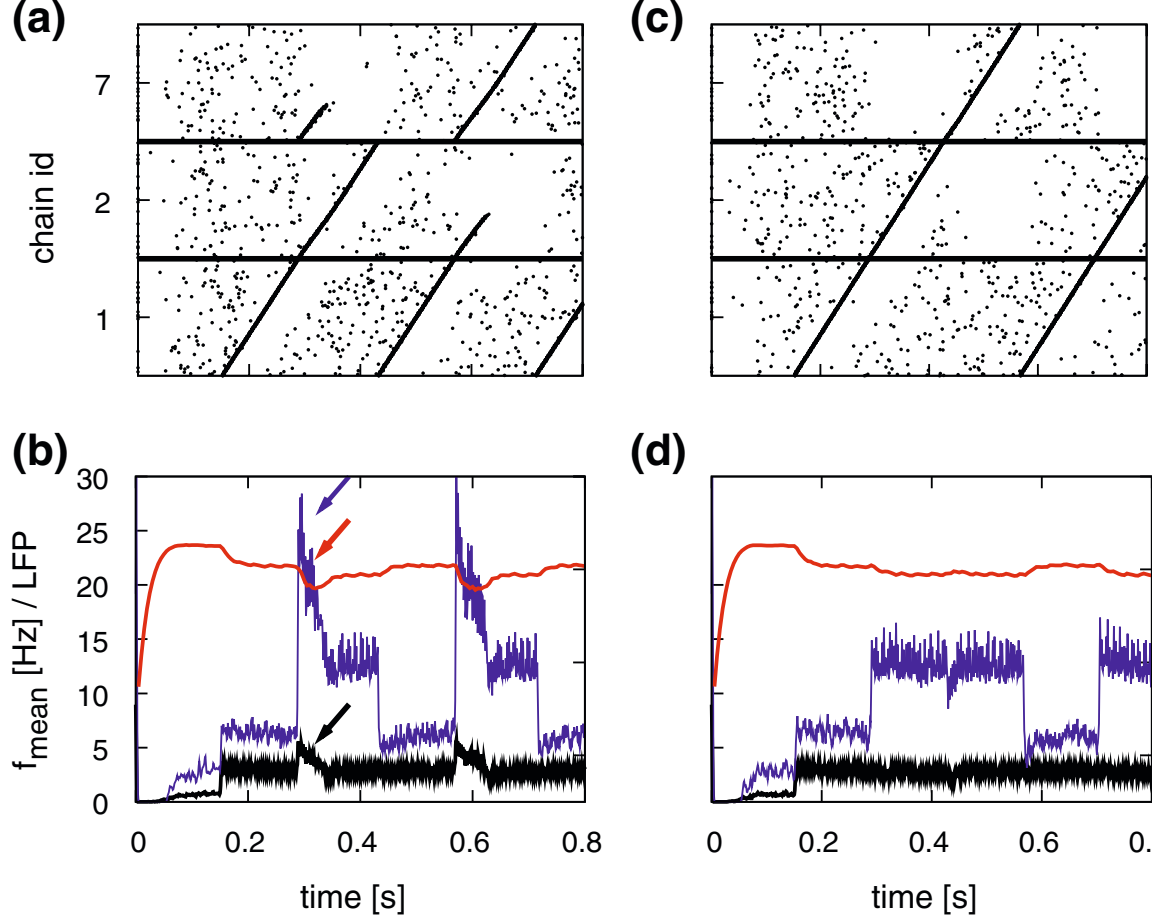

(d)

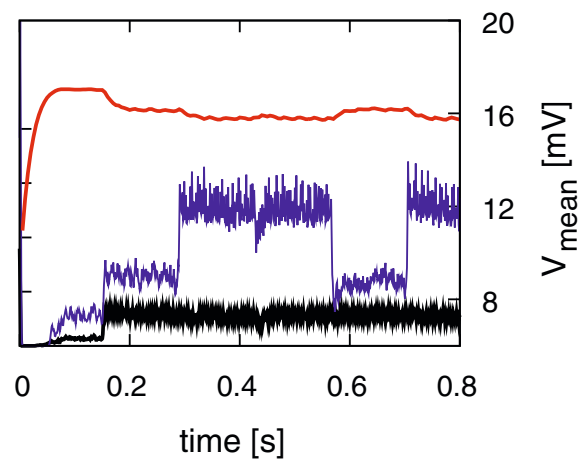


of generating the two types of switching errors for the network as functions of $k_{\mathrm{c}}$ and $k_{\mathrm{g}}$. Each configuration is stimulated 100 times by applying a Gaussian pulse packet to the initial pool of chain 1 . As the number of unstructured cross-inhibition connections $k_{\mathrm{c}}$ increases, the probability of activating both successor chains decreases (Fig. 11(a)) whilst the probability that neither chain is activated increases (Fig. 11(b)). The influence of $k_{\mathrm{g}}$ on the switching errors is less pronounced but acts in the same direction as $k_{\mathrm{c}}$. A good choice for the number of inhibitory connections is $k_{\mathrm{c}}=19$ and $k_{\mathrm{g}}=$ 7: for this configuration, the probability of activating both successor chains is $p_{2}=0 \%$, whereas the probability that neither chain is activated is $p_{0}=4.51 \% \pm$ 2.66. As before, the means and standard deviations for these two values are calculated by performing the 100 switching trials on 100 different network realizations. This configuration exhibits a reasonable compromise between reliable switching and stability and is used for the rest of this manuscript. As in the case of structured cross-inhibition, for a given realization of the network a bias towards selecting chain 2 or chain 7 can be observed, however on average there is no bias due to the symmetry of the connection distribution (data not shown).

\subsubsection{Competition between multiple chains}

To generate a series of parabolic segments, it is necessary that the network enables reliable switching from one chain to exactly one of its successor chains. The (a)

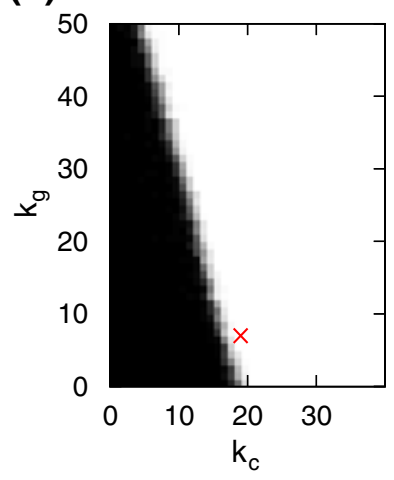

(b)

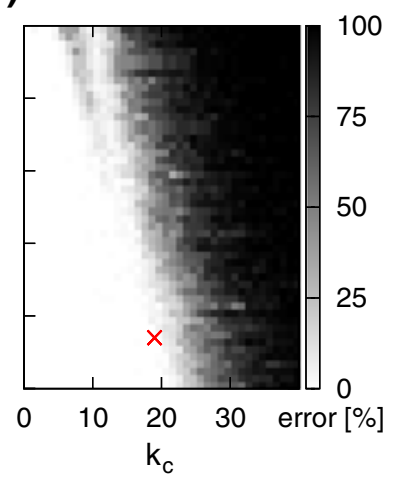

Fig. 11 Switching errors as a function of the number of inhibitory cross connections $k_{\mathrm{c}}$ (horizontal axis) and the number of global inhibitory connections $k_{\mathrm{g}}$ (vertical axis). (a) Percentage of trials resulting in the activation of both successor chains (b) Percentage of trials resulting in the activation of neither of the successor chains. In both plots the red cross indicates the chosen parameter set $k_{\mathrm{c}}=19$ and $k_{\mathrm{g}}=7$ robust winner-takes-all mechanism proposed by Chang and Jin (2009) cannot be realized, as it depends on dominant global inhibition, whereas our network operates in the asynchronous irregular regime which entails a balance of excitation and inhibition (Brunel 2000). However, in Sections 3.2.1 and 3.2.2 we showed that switching to one of two possible successor chains is reliable if the inhibition strength and connectivity are chosen appropriately. Here we investigate whether competition by mutual inhibition can be extended to the case of more than two successor chains. Figure 12(a, b) shows the total error probabilities for structured and unstructured cross-inhibition when switching to one of two successor chains, i.e. the sum of the number of trials in which more than one successor chain was activated and the number of trials in which no successor chain was activated. The working regime for structured crossinhibition is much smaller than that for unstructured cross-inhibition. We therefore investigate the total error probability for three and four successor chains assuming unstructured cross-inhibition, see Fig. 12(c, d). The working regime shrinks with increasing number of successor chains.

However, in most natural movement scenarios the choice of the next action is unlikely to be truly random, but influenced by the existence of imprinted repetitive movement sequences or by priming from motor planning and control areas. In order to illustrate the effect of a very simple priming mechanism on the reliability of switching, we apply an additional Poisson input of $1 \mathrm{kHz}$ with variable synaptic weight $J_{\text {prim }}$ to all neurons of the first 10 pools of one successor (chain 2) in a network with four potential successor chains. The inhibition parameters are chosen as $k_{\mathrm{c}}=25$ and $k_{\mathrm{g}}=7$ (see red cross in Fig. 12(d)). The conditional probability of switching to chain 2 , given a correct switch to exactly one of the successor chains, is shown in Fig. 12(e) as a function of $J_{\text {prim. }}$. The conditional probability of switching to chain 2 increases with increasing excitatory priming and decreases with increasing inhibitory priming. It can be fitted by a sigmoidal function $(f(x)=$ $100 /\left(1+e^{-a(x+b)}\right)$ where $a=1.8$ and $\left.b=0.4\right)$.

The total error probability decreases with increasing excitatory or inhibitory priming and is maximal when the network is operating at chance level, i.e. the probability of switching to any of the four successor chains is $25 \%$. The chance level shown in Fig. 12(e) is estimated from the fitted function and is slightly shifted from the unprimed case towards inhibitory priming with $J_{\text {prim }}=-0.98 \mathrm{pA}$. This is due to the random network connectivity which results in minor asymmetries in the switching probabilities. Specifically, the probability that 
(a)

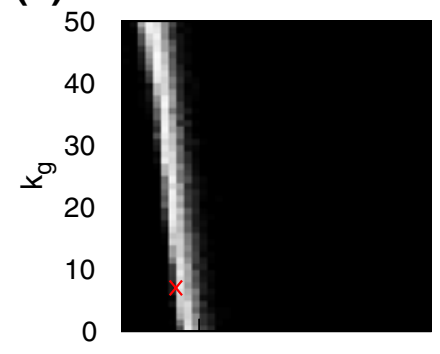

(b)

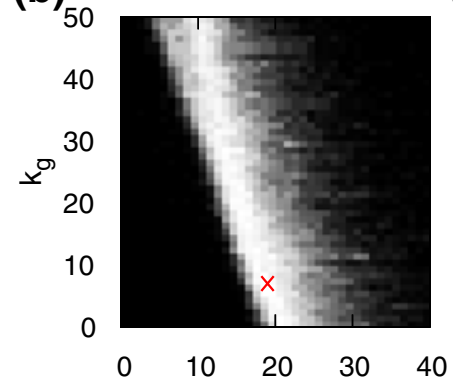

(c)

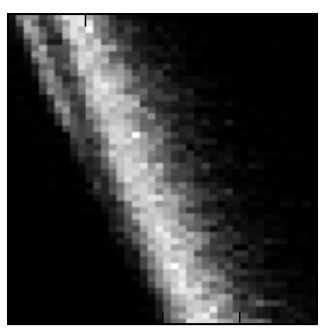

(d)

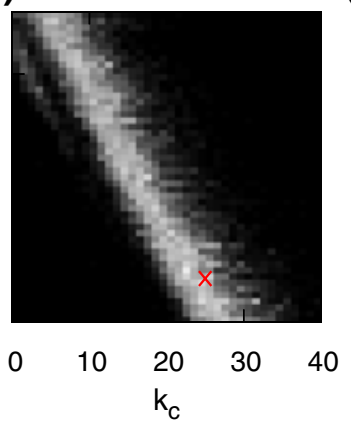

(e)

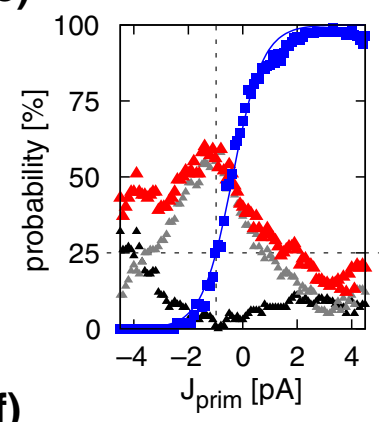

(f)

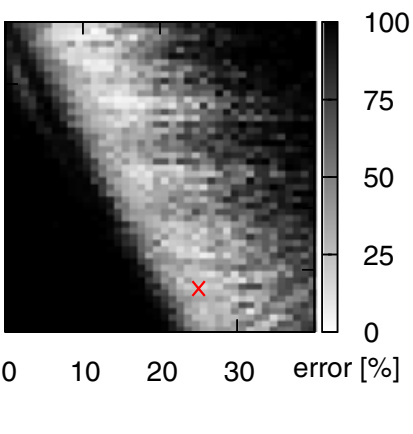

Fig. 12 Total probability of switching errors as a function of the number of successor chains. (a) Total probability of switching error for two potential successor chains assuming structured cross-inhibition as a function of the number of inhibitory cross connections $k_{\mathrm{c}}$ and number of global inhibitory connections $k_{\mathrm{g}}$. The plot shows the summed probabilities of Fig. 8(a) (probability of activating more than one successor chain) and Fig. 8(b) (probability of activating no successor chain). The red cross marks the parameter set $k_{\mathrm{c}}=k_{g}=7$. (b) As for (a) for the case of unstructured cross-inhibition; the red cross marks the parameter set $k_{\mathrm{c}}=19$ and $k_{\mathrm{g}}=7$. (c) As for (b) but for three potential successor chains. (d) As for (b) but for four potential successor chains; the red cross marks $k_{\mathrm{c}}=25$ and $k_{\mathrm{g}}=7$. (e) Conditional probability of switching to chain 2 as a function of the priming strength $J_{\text {prim }}$ for $k_{\mathrm{c}}=25$ and $k_{\mathrm{g}}=7$ (blue squares; sigmoidal fit to the data, blue curve). Probability of activating no successor chains (grey triangles), probability of activating multiple successor chains (black triangles) and total switching errors (red triangles). Dotted lines indicate chance level, i.e. a $25 \%$ probability of activating chain 2. (f) As for (d) but with excitatory priming $J_{\text {prim }}=3$ pA to chain 2 no successor chain is chosen is maximal at chance level as the symmetry between the activity in all potential successor chains increases the likelihood that no winner emerges. Applying inhibitory or excitatory priming introduces a bias into the competition, thus making it more likely that activity will propagate in at least one chain. Conversely, the probability that multiple chains are activated is approximately constant for excitatory priming and increases with increasing inhibitory priming. This effect is due to the general reduction of activity in chain 2 , which results in less global inhibition in the network and thus a greater probability of activity propagating in more than one chain (see also Fig. 11(a)). At around $J_{\text {prim }}>4 \mathrm{pA}$ the general increase of activity in chain 2 enables the spontaneous triggering of synfire activity (Tetzlaff et al. 2002). In this network regime, reliable switching is no longer possible and so the total error probability increases. Fig. 12(f) shows the case of four chain switching with excitatory priming to chain $2\left(J_{\text {prim }}=3 \mathrm{pA}\right)$. The introduction of prim-

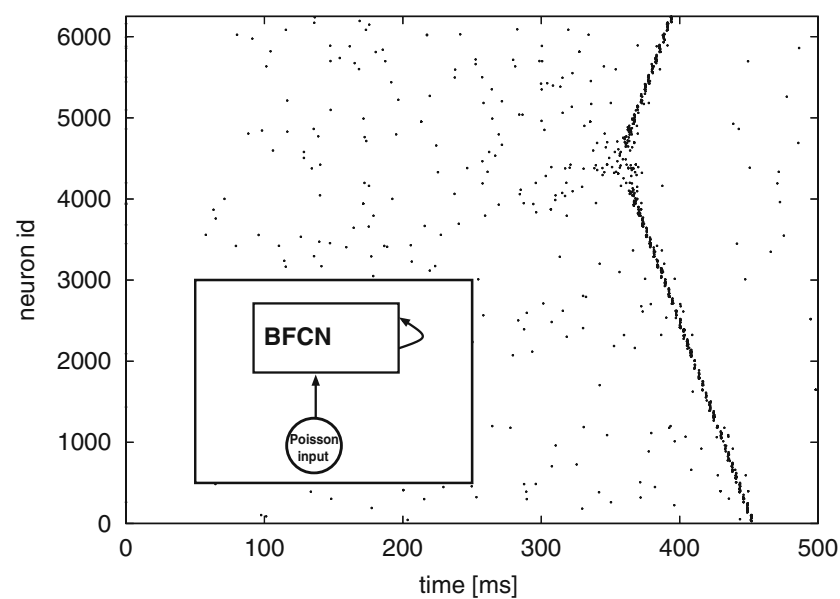

Fig. 13 Spontaneous synfire activity in the BFCN without global inhibition $k_{\mathrm{g}}^{*}=0$ and with an external excitatory Poissonian input of $f_{\mathrm{ex}}=7.7 \mathrm{kHz}$ to each neuron (see inset). The activity of $10 \%$ of the neurons is shown 
Fig. 14 Activity state transition in the BFCN with global inhibition, network set-up top right. (a) Spiking activity in the BFCN plotted with an external excitatory Poissonian input of $f_{\text {ex }}=7.65 \mathrm{kHz}$ to each neuron and $k_{\mathrm{g}}^{*}=7$. Activity of $10 \%$ of the neurons is shown. (b) As in (a) but for $f_{\mathrm{ex}}=7.9 \mathrm{kHz}(\mathbf{c})$ Oscillation frequency of synfire waves as a function of the rate of the external Poisson input for different values of global inhibition: $k_{\mathrm{g}}^{*}=0$ (black squares), $k_{\mathrm{g}}^{*}=7$ (dark gray disks), $k_{\mathrm{g}}^{*}=21$ (light gray triangles)
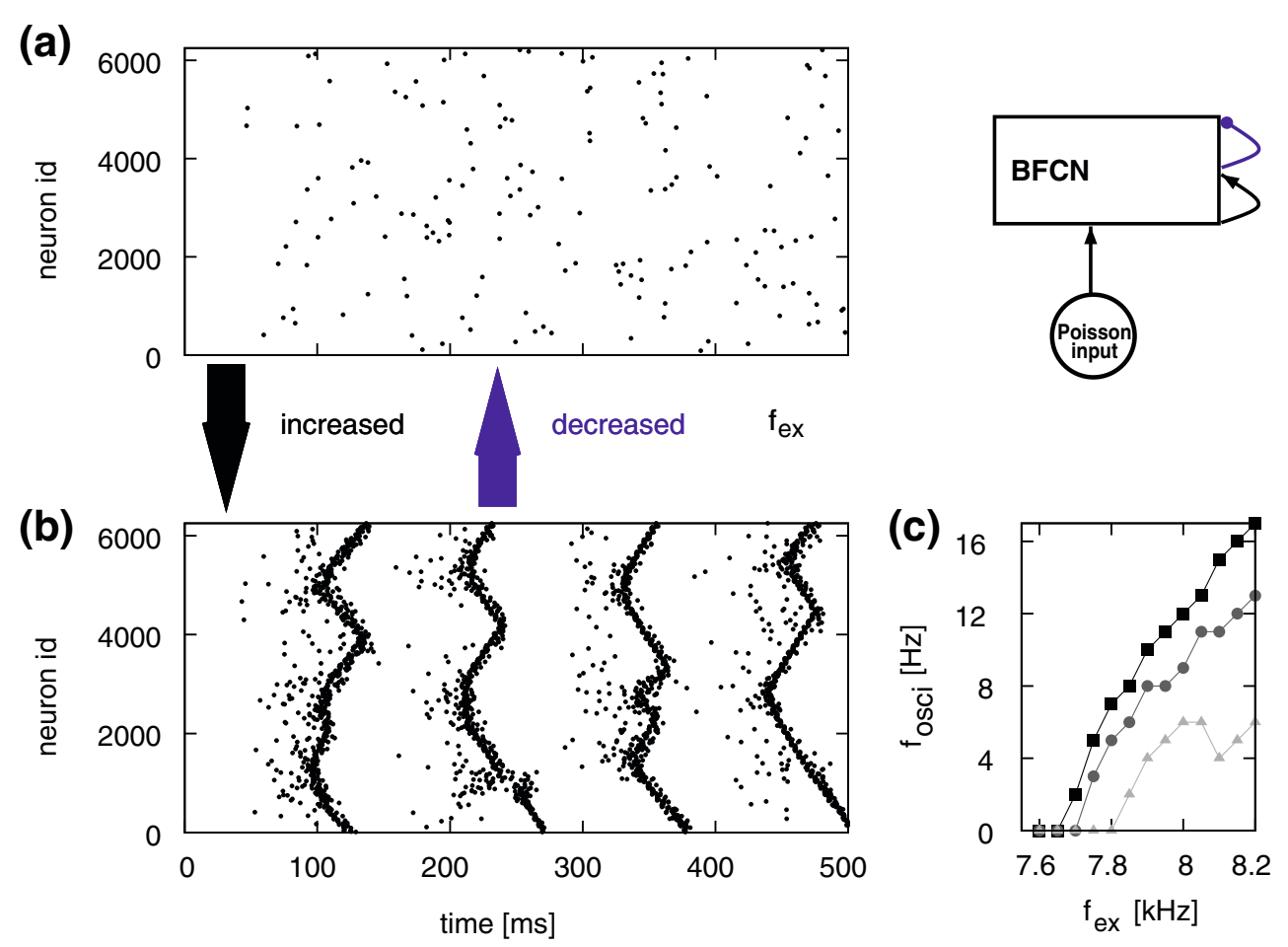

ing results in a large increase of the working regime (compare Fig. 12(d)). For the rest of the manuscript we restrict our analysis to the case of two successor chains with no priming, as this is sufficient to model the key features of the behavior of a highly trained and motivated monkey in the free scribbling task.

\subsection{Activity state transition in the BFC}

When the backward-and-forward connected chain network (BFCN) described in Section 3.1 is not being inhibited by the presence of synfire chain activity in the synfire chain network (SFCN), the external drive is just strong enough to induce spontaneous synfire activity and so re-ignite activity in the SFCN. The ignition of synfire activity in the network can be understood intuitively as follows: random synchronous activity in a small subset of the neurons in a given pool $i$ will be projected to the pools $i \pm 1$, which in turn project back to $i$, thus building a recurrent positive feedback loop. In the example given in Fig. 13, synfire activity emerges spontaneously at around $380 \mathrm{~ms}$ and propagates in both directions along the chain. Once the spike volleys have reached the ends of the BFC the synfire activity is extinguished. A reflection of activity does not occur because for any activated pool, the neurons in the previously activated pool have been reset by the propagating volley. When the SFCN has been re-ignited, the increased inhibition decreases the net drive to the network such that no spontaneous synfire activity occurs.

(a)

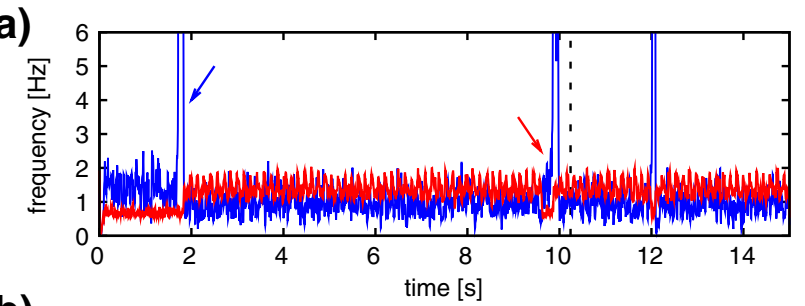

(b)

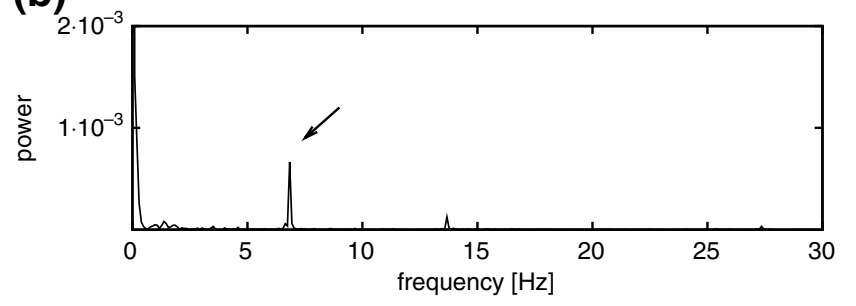

Fig. 15 Sustained activity in the coupled network: (a) Gaussian filtered $(\sigma=5 \mathrm{~ms})$ firing rate of the SFCN (red) and the BFCN (blue) as functions of time. Activity of $10 \%$ of the neurons is recorded. The blue arrow indicates a spontaneous emergence of synfire activity in the BFCN, resulting in a substantial transient increase in firing rate. The red arrow highlights a switching failure in which neither successor chain is activated, causing activity in the SFCN to die out temporarily. (b) Power spectrum of the average Gaussian filtered firing rate between 0 and $10.24 \mathrm{~s}$ (vertical black dashed line in (a)). Black arrow indicates peak at around $7 \mathrm{~Hz}$ 
The activity transition in the BFCN can be seen with greater clarity by investigating a more general version of the network with non-zero global inhibition $\left(k_{\mathrm{g}}^{*}>0\right)$ which balances the network activity and a variable strength of external drive. An example with $k_{\mathrm{g}}^{*}=7$ is given in Fig. 14(a) and (b). If the external drive is chosen below the transition point $\left(f_{\mathrm{ex}}=7.65 \mathrm{kHz}\right)$ the network exhibits low rate asynchronous activity and no spontaneous synfire activity. This is illustrated in Fig. 14(a). If the external drive is chosen above the transition point $\left(f_{\mathrm{ex}}=7.9 \mathrm{kHz}\right.$ in Fig. $\left.14(\mathrm{~b})\right)$, the network exhibits periodic waves of synfire activity. The oscillation frequency of the synfire activity increases with increasing external drive $f_{\mathrm{ex}}$ and the transition point shifts to higher values of $f_{\text {ex }}$ with increased global inhibition (see Fig. 14(c)).

As can be seen in Fig. 14(b), synfire volleys caused by spontaneous self-ignition tend to be of limited duration. Activity volleys traveling in different directions cancel each other when they meet and volleys reaching either end of the BFCN are not reflected. Furthermore, the high activity in the BFCN during synfire activity results in strong global inhibition and a reset of nearly all neurons, which in turn decreases the probability of self-ignition until activity has built up again. However, if the pool size is chosen sufficiently large (e.g. 175 neurons for $k_{\mathrm{g}}^{*}=0$ and $f_{\mathrm{ex}}=7.7 \mathrm{kHz}$ ), a single selfignition results in ongoing pathological high firing rates.

\subsection{Sustained activity}

We exploit the self-igniting property of the BFCN to sustain activity in the SFCN described in Section 3.1 and illustrated in Fig. 5. Inhibitory connections from the SFCN suppress the activity of the BFCN when synfire activity is present, as this is characterized by a high firing rate. When no synfire activity is present in the SFCN, it fires in the asynchronous irregular regime at low rate and consequently is not able to suppress the activity in the BFCN. An example is given in Fig. 15(a). In the beginning no synfire activity is present in the SFCN and the BFCN is not suppressed. At around $1.8 \mathrm{~s}$ the BFCN self-ignites as described in Section 3.3, leading to a sharp increase in its firing rate. The BFCN activates chain 1 in the SFCN. The subsequent synfire activity suppresses the BFCN activity below the selfignition threshold. The synfire activity lasts for $8 \mathrm{~s}$ before the activity dies away due to a switching error. The inhibitory input to the BFCN decreases as the SFCN firing rate drops, allowing the BFCN activity to rise above the self-ignition threshold again. At around $9.8 \mathrm{~s}$
Fig. 16 Generation of scribbling trajectories. (a) Spiking activity of BFCN and SFCN. Activity of $1 \%$ of the neurons is shown. The activity of each synfire chain is plotted in a strip marked by horizontal lines in the color of the corresponding arrow in velocity space shown in (b). $\mathrm{BFC}$ activity (blue) is plotted in the top strip. Above the raster plot the average firing rate of the SFCN (red) and the $\mathrm{BFCN}$ (blue) is plotted. (b) Reproduction of Fig. 4 for ease of reference. (c) Scribbling trajectory extracted from the spiking activity using population coding. Segments are drawn in the color of the most active chain

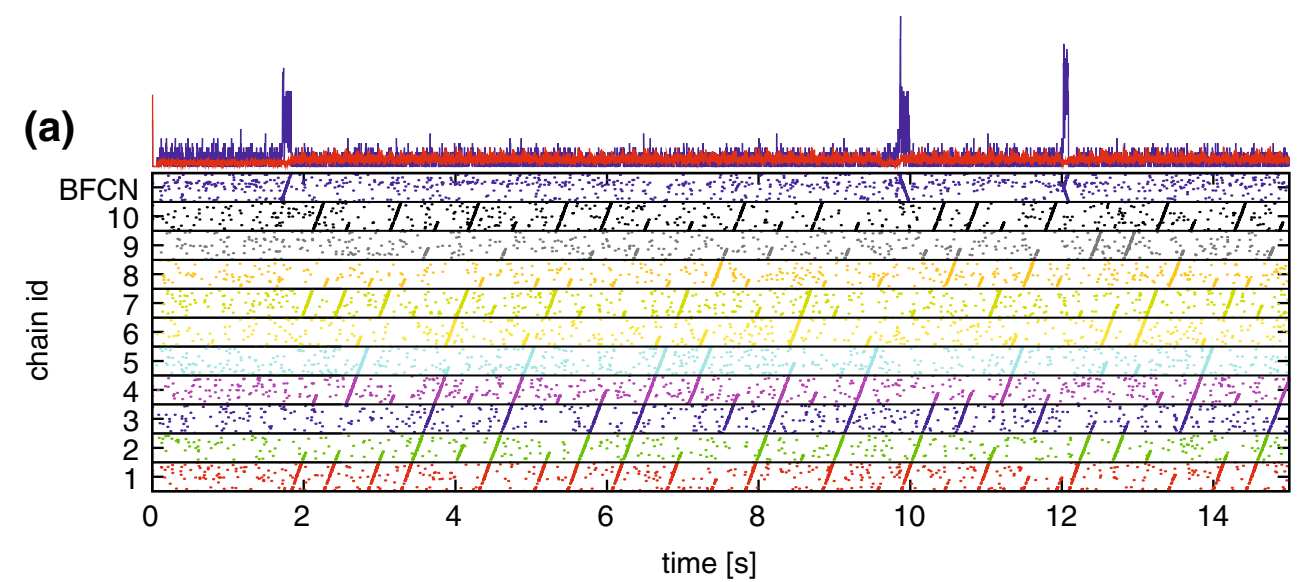

(b)

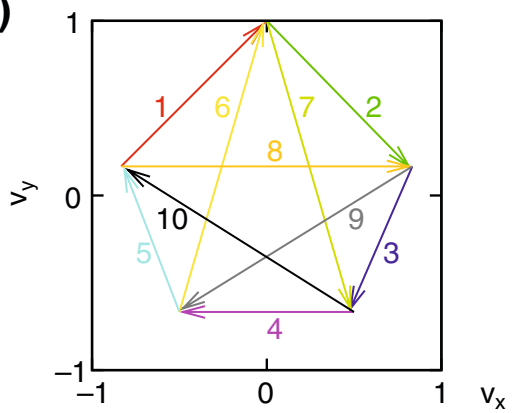

(c)

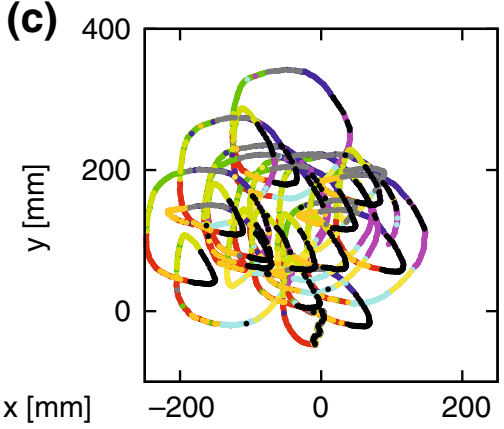


Fig. 17 Equi-affine analysis of the generated trajectory. (a) Section of spike data shown in Fig. 16(a). Vertical dashed lines indicate the extent of individual synfire chain activities. (b) First section of the scribbling trajectory shown in Fig. 16(c), generated between $1.7 \mathrm{~s}$ and $2.7 \mathrm{~s}$.

(b) Velocity of the generated trajectory in the $x$ (black) and $y$ (gray) directions as functions of time. (c) Acceleration of the generated trajectory in the $x$ (black) and $y$ (gray) directions as functions of time. (d)

Equi-affine curvature $\kappa$ of the generated trajectory as a function of time. Colored vertical lines indicate the start of the corresponding parabolic primitive (a)

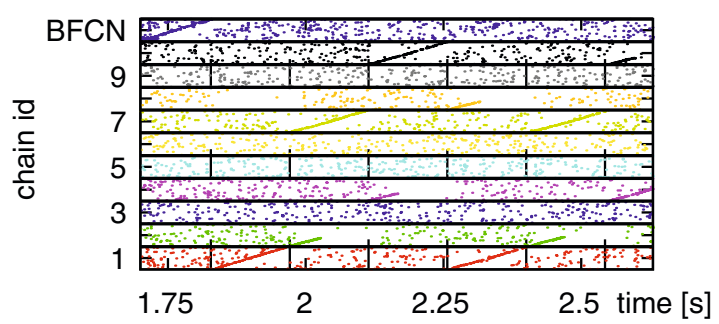

(b)

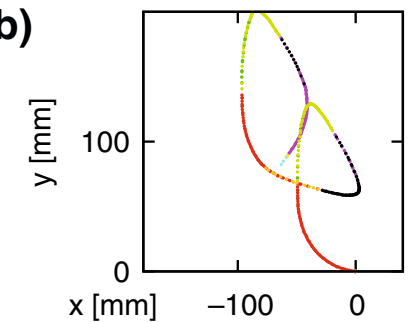

(c)

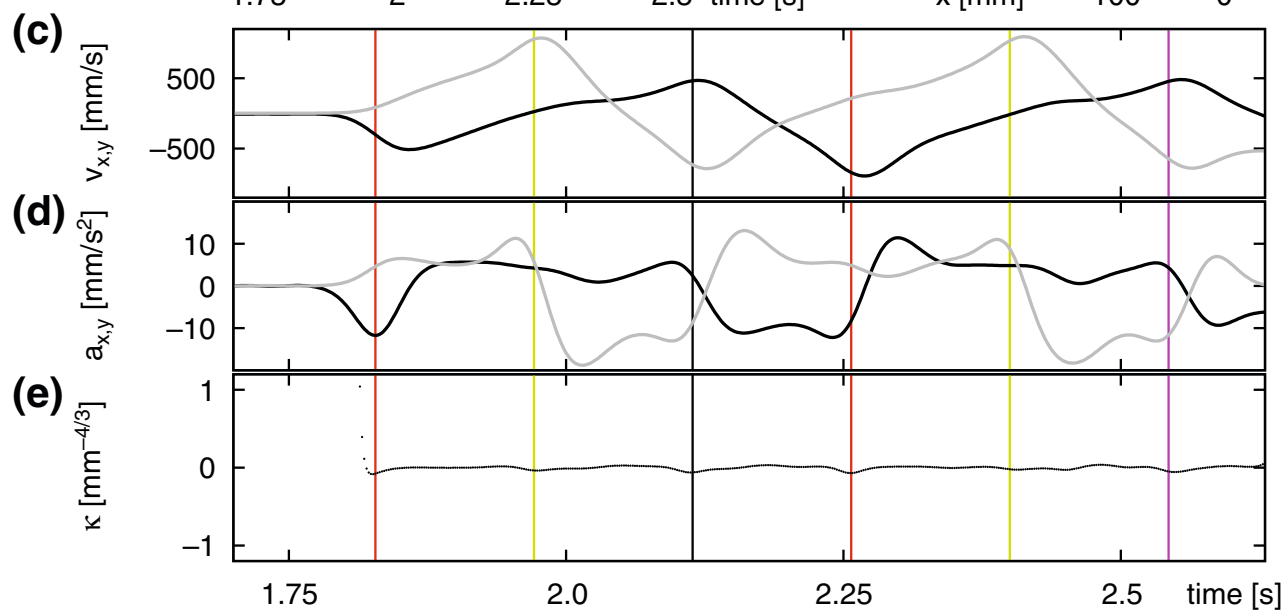

the BFCN self-ignites again, inducing a fresh sequence of SFCN activity. The low frequency signature of the synfire chain competition discussed in Section 3.2 can be clearly seen in the full network as a peak at around $7 \mathrm{~Hz}$ in the Fourier transformed firing rate shown in Fig. 15(b). This is the expected frequency because the synfire activity duration in each chain is approximately 140 ms (see Fig. 3).

\subsection{Generation of scribbling trajectories}

The spiking activity of the complete network underlying the average firing rates shown in Fig. 15 is given in Fig. 16(a). The trajectory extracted from the spiking activity as described in Section 2.3 is shown in Fig. 16(c). The trajectory consists of a long random sequence of parabolic movement primitives. Small overlaps can be seen at the transition points where both successor chains are active before one of the chains wins the competition. The distribution of the length $n$ of an uninterrupted sequence is well fitted by $P(n)=p_{0}\left(1-p_{0}\right)^{n}$, where $p_{0}$ is the probability that neither successor chain is activated during synfire chain switching (data not shown).

We analyze the characteristics of the trajectory shown in Fig. 16(c). In Fig. 17(b) the first part of the trajectory is displayed in the colors of the most active synfire chain as calculated from the spiking activity in Fig. 17(a). For increased clarity in Fig. 17(c-e), colored vertical lines indicate transitions to the corresponding parabolic segments as identified in Fig. 17(b). The velocity of the trajectory in the $x$ and $y$ directions is extracted from the spiking activity by calculating the population coding in $2 \mathrm{~ms}$ bins and smoothing with a Gaussian kernel with standard deviation $\sigma=10 \mathrm{~ms}$. Figure 17(c) shows that the velocities vary approximately linearly during the activity time of a given chain. This can also be seen by considering the accelerations in the $x$ and $y$ directions, which are calculated using the finite difference method between successive sample points of the velocities and shown in Fig. 17(d). The accelerations are approximately constant during the activity time of a given chain. Due to the piecewise constant accelerations, following the derivation in Section 2.1 we conclude that the trajectory does indeed fulfil the two-thirds power law (e.g. Viviani and Flash 1995). Figure 17(e) shows that the equi-affine curvature of the trajectory is close to zero (see Section 2.5 for details of analysis). We therefore conclude that the trajectory does indeed consist of a series of parabolic segments.

\section{Discussion}

In this study we have demonstrated that activity in synfire chains can realize the generation of experimentally observed parabolic segments that fulfill the 
two-thirds power law (Viviani and Flash 1995; Polyakov et al. 2009b). The key insights are that uniform linear motion in velocity space maps to parabolic motion in position space and that the propagation of a wave of activity in a synfire chain is an ideal neural mechanism for a process characterized by uniform linear motion. We further show that a network of synfire chains can produce on-going trajectories consisting of series of parabolic segments with smooth transitions among them. Necessary assumptions for this to happen are that the terminal pools of the synfire chains have a similar preferred velocity as the initial pools of the chains to which they connect to, and that the switching mechanism that selects an appropriate successor chain is reliable. A final aspect of the model is that the extinction of synfire activity triggers a mechanism by which it is re-ignited. In summary, by postulating an appropriate structure in a simulated network of biologically realistic neurons we have developed a model that reliably reproduces macroscopic properties of monkey scribbling. In the following sections we discuss the assumptions, limits and predictions of our model in greater detail.

\subsection{Synfire chains}

The choice of synfire chains as the chief computational element of the model results from a possible interpretation of the two-thirds power law as a linear propagation in velocity space. While other interpretations of the two-thirds power law are conceivable, the simplicity of the present assumption is not only theoretically appealing, but is also a good match to the known properties of synfire chains in maintaining an accurate representation of a constant progression (Ha $\beta$ et al. 2008). The assumption of synfire chains as constituents of the model is also in good agreement with Fitts' law, since the speed of the synfire activity can be reliably controlled, for example by the variation of global noise or by the modulation of neural threshold values (Wennekers and Palm 1996). The example studied here considers only five basic speeds and their one-parametric linear combinations. The model is obviously generalizable to a larger number of accelerating forces to the arm provided that the matching conditions between terminal speed of one chain and the initial speed of another one are observed. Even a continuous arrangement of synfire structures in form of a two-dimensional neural field (Amari 1977) is imaginable, but it should be noted that the data (Polyakov et al. 2009a, b) are largely representable by as little as three unidirectional chains. This impoverishment of the movement manifold might be due to continued optimization under constant experimental conditions and can be assumed to reflect a compromise between the goal of the task and the energy-efficient control of the arm. Thus, we might speculate that even in a more general framework, certain movements would be preferred to others. Consequently, a discrete representation, save for some extraneously encoded invariances, might be sufficient to account for planned optimized movements of the extremities.

\subsection{Competition between synfire chains}

We investigated two alternate switching mechanisms on the basis of mutual cross-inhibition (see Section 3.2). We have shown that both structured and unstructured cross-inhibition realize reliable switching, but that unstructured cross-inhibition is more effective at eliminating the occurrence of two successor chains being activated without substantially increasing the risk of activity dying away altogether. Additionally, the working regime in which reliable switching can be achieved is greater for unstructured than for structured crossinhibition. Note that in this study, each inhibitory neuron has the same number of global and the same number of local outgoing connections. A finer tuning could be achieved by assuming that the numbers of outgoing inhibitory connections are drawn from distributions or not homogeneous along the length of a chain. The question of whether the assumption of structured or unstructured cross-inhibition is more realistic in the motor cortex cannot be answered with the experimental data currently available. In Section 3.2.3 we investigated the scaling properties of the switching mechanism by unstructured mutual inhibition in networks with greater numbers of potential successors. The working regime shrinks with increasing number of successor chains, however, even a very simple priming mechanism restores the working regime.

The two architectures investigated here are not the only possible candidates for a reliable switching mechanism. For example, an alternative architecture realizing synfire chain competition on the basis of a dominant global inhibition was recently proposed by Chang and Jin (2009). Their simplified model, with pulse coupling (i.e. zero delays) and constant superthreshold drive to the individual neurons ensures that only one neuron is active at a time (Jin 2002). In this case the robustness of the switching can be shown analytically. In a subsequent study, Jin (2009) showed that their proposed mechanism also enables robust synfire chain switching in a biologically realistic model of songbird HVC (high vocal center) nuclei. However, syllable-coding 
excitatory neurons in HVC exhibit sparse bursting behavior (Hahnloser et al. 2002) while the inhibitory interneurons fire with high frequencies (Dutar et al. 1998). This activity regime differs markedly from that of the motor cortex investigated in our model, which is characterized by on-going asynchronous irregular activity.

\subsection{Ignition of synfire activity}

Our model makes use of a backward-and-forward connected chain to ignite synfire chain activity in the SFCN at the beginning of the simulation and after switching failures cause the synfire activity to die out. We have characterized the dynamical properties of the BFCN, such as periodic ignition and self-extinction of synfire activity. The choice of the BFCN is not crucial for our model; clearly, a variety of network architectures could fulfil its role. The critical mechanism is the existence of an inhibitory/excitatory reciprocal connection between the networks, such that synfire activity in the SFCN inhibits activity in the other network, which in turn excites the SFCN when the inhibition is reduced. The advantage of the BFCN is that it creates a pulse packet of exactly the right width and amplitude to guarantee that synfire activity is triggered in the SFCN. We therefore include it to demonstrate that changes in the standard synfire chain architecture can lead to interesting functional properties that have not previously been investigated. For the sake of simplicity we restricted the model to a single BFC which can only ignite the first chain; in this study we are investigating a possible neuronal architecture that could underlie the observed segmentation of 2D movement trajectories, rather than the characteristics of movement initialization after a still period. However, the model could be extended to include several BFCs projecting to different first pools in order to investigate this aspect of behavior. The interconnection between BFCs and directional synfire chains can be learned by a realistic Hebbian rule as described in $\mathrm{Ha} \beta$ et al. (2008) for a somewhat simpler model.

\subsection{Learning and synfire chains}

Our model is predicated on the existence of synfire chains. Many studies have reported precise spike timing, for example in mammalian cortex (Eckhorn et al. 1988; Gray and Singer 1989; Prut et al. 1998; Abeles et al. 1993; Ikegaya et al. 2004; Pulvermüeller and Shtyrov 2009) or songbird HVC (Hahnloser et al. 2002;
Kozhevnikov and Fee 2007), which suggests an underlying feed-forward connectivity. Moreover, a local convergent-divergent connectivity profile has been experimentally observed in HVC (Mooney and Prather 2005). It has also been shown experimentally that the learning of new motor tasks leads to changes in synaptic strength and the rapid formation of new synaptic connections (Rioult-Pedotti et al. 1998; Xu et al. 2009), however there is as yet no consensus on the mechanisms required for the brain to develop such structures. Although some modeling studies have reported the development of feed-forward sub-networks (Izhikevich et al. 2004; Buonomano 2005; Doursat and Bienenstock 2006; Jun and Jin 2007; Fiete et al. 2010) on the basis of Hebbian synaptic plasticity, studies of large-scale networks with biologically realistic numbers of synapses per neuron have not reproduced these findings (Morrison et al. 2007a; Kunkel et al. 2010). This highlights the need for further studies to determine the necessary and sufficient conditions for the divergentconvergent connectivity of synfire chains to develop.

The introduction of synaptic plasticity could also address another limitation of our model, namely that the transition probabilities between one primitive and the next are constant. Hebbian plasticity at the transitions between one chain and the next would imprint trajectories that are carried out more often (Hanuschkin et al. 2010a) whereas reward-modulated plasticity would imprint trajectories that are more likely to generate a reward (e.g. Izhikevich 2007; Farries and Fairhall 2007; Baras and Meir 2007; Legenstein et al. 2008; Potjans et al. 2009, 2010). As shown in Section 3.2.3 the probability of a switching failure increase with the number of successors. A reward can be externally applied, but it is also conceivable that the increase in the network rate caused when more than one potential successor chain is activated, or the rate decrease when no successor chain is activated, could be interpreted by the network as a negative reward signal. The effect of the rewardmodulated plasticity in this case would be to decrease the variability (number of possible successors) in generated motor sequences.

The decision where to continue after reaching the end of a synfire chain or, considered here as equivalent, a movement primitive, might be biased by a number of factors such as movement intentions, visual feedback or biomechanical constraints. An alternative approach to generate specific rather than random trajectories is therefore to influence the switching mechanism by priming one potential successor chain, as investigated in Section 3.2.3. The priming signals can be generated by an additional network (Hanuschkin et al. 2010b) or even within the same network if neural populations 
with different time constants are assumed (Yamashita and Tani 2008). Experiments suggest that sequential information and directional coding are indeed coded within the same neuronal population (Carpenter et al. 1999; Ben-Shaul et al. 2004). A repeated application of priming to generate a specific movement sequence could induce the imprinting of the sequence through the Hebbian mechanisms discussed above, rendering future priming unnecessary. On a behavioral level this can be interpreted as the convergence of a motor pattern from a consciously controlled variable to a fully automatic execution of a stereotyped movement after intensive training (Sforza et al. 2000).

\subsection{Predictions of the model}

In our model study of free monkey scribbling we show that cell assembly competition generates low frequency oscillations in collective signals that approximate the LFP. In our simulations, all synfire chains are the same length and generate primitives with durations of about $140 \mathrm{~ms}$, leading to competition-driven oscillations in the LFP at around $7 \mathrm{~Hz}$. In experiments the median drawing time of a parabolic movement is $250 \mathrm{~ms}$; our model therefore predicts competition-driven oscillations in the LFP around $4 \mathrm{~Hz}$. This component is unlikely to be as well-defined as in our simplified model, as feedforward structures embedded in real cortical tissue are likely to be overlapping and of varying lengths, masking the effect of low LFP oscillations. Additionally, when the switching is biased due to priming or learned asymmetries in the connections to potential successor chains, the degree of competition is lowered. This suggests that the low frequency component of the LFP will decrease as training progresses, as stereotypical movements are imprinted or priming is activated. Experimental studies have shown that the low frequency LFP $(<13 \mathrm{~Hz})$ is suitable for extracting motion direction of stereotyped movements by investigating the movement evoked potential (mEP) (Rickert et al. 2005; O'Leary and Hatsopoulos 2006), but so far studies investigating oscillations during continuous movements have focussed on the beta to gamma band $(\sim 15-90 \mathrm{~Hz})$ rather than the low frequency band (Donoghue et al. 1998). In our model the approximated LFP signal contains no information about the direction of movement, as the neurons are not spatially organized and each neuron contributes equally to the signal. However, in future studies this simplification could be relaxed in order to take the spatial extent of an LFP signal into consideration, as could the high degree of symmetry which is unlikely to occur in a biological system.
Along with the effect of introducing low frequency components to the LFP, competition between cell assemblies also produces deviations from the pure parabolic trajectories at the transition sites between movement primitives. This effect can indeed also be seen in the analysis of the experimental movement trajectories: in Fig. 4(a) of Polyakov et al. (2009b), deviations from the $\kappa=0$ line can be observed that may indicate transition points between movement primitives. Our model predicts that these deflections will decrease with training, as training reduces the competition by priming or imprinting the sequence of cell assemblies. As our model does not include any priming or learning during the generation of movement sequences, the length of the trajectories follows the geometric distribution $P(n)=p_{0}\left(1-p_{0}\right)^{n}$, where $p_{0}$ is the constant probability that no successor chain is activated at a switching point (Section 3.5). This distribution is unlikely to occur in real experimental data, as a real monkey will exhibit varying levels of motivation during a trial. However, our experiments on priming suggest that the mean length of trajectories will increase with training, as the transitions from one primitive to the next become more reliable due to priming of the successor chain or strengthening of the transition connections (imprinting). To verify these predictions, further experimental studies on the development of the equi-affine properties and characteristic lengths of on-going trajectories with training could be carried out.

A final prediction of the model is the existence of directionally un-tuned neurons in the motor cortex that increase their firing rate immediately before movement onset (see Fig. 15). A possible signature of this population activity is the peak in the mEP observed just before movement onset (Rickert et al. 2005). Additional evidence to support this interpretation is that the peak amplitude decreases when the monkey can anticipate the movement to execute (Roux et al. 2006). In our model, this would correspond to inducing a new movement primitive through a priming signal rather than through ignition. To investigate these issues further, more sophisticated theoretical models of the generation of the LFP in a simulated network are required (Lindén et al. 2009a, b). Instead of high frequency oscillations serving to bind distributed cortical representations as proposed by Singer and Gray (1995), in our study composition is expressed in low frequency oscillations resulting from cell assembly interaction. We therefore suggest greater attention should be paid to low frequency components in experimental data and that data sets recorded from partially trained animals may be particularly helpful in revealing the mechanisms of motor compositionality. 
Acknowledgements Partially funded by DIP F1.2, BMBF Grants 01GQ0420/01GQ0432 to BCCNs Freiburg and Göttingen, EU Grant 15879 (FACETS), Helmholtz Alliance on Systems Biology (Germany), Next-Generation Supercomputer Project of MEXT (Japan) and the Junior Professor Program of Baden-Württemberg. The authors would like to cordially thank the members of the DIP F1.2 collaboration for their always stimulating and constructive discussions of this work, in particular T. Flash, F. Polyakov, M. Abeles, M. Teicher and T. Geisel. Finally, we would like to thank S. Schrader for his support at the outset of this project and M. Denker for useful comments on an earlier version of the manuscript.

Open Access This article is distributed under the terms of the Creative Commons Attribution Noncommercial License which permits any noncommercial use, distribution, and reproduction in any medium, provided the original author(s) and source are credited.

\section{References}

Abbott, L. F. (1999). Lapicque's introduction of the integrateand-fire model neuron (1907). Brain Research Bulletin, 50 (5-6), 303-304.

Abeles, M. (1991). Corticonics: Neural circuits of the cerebral cortex (1st ed.). Cambridge: Cambridge University Press.

Abeles, M., Bergman, H., Margalit, E., \& Vaadia, E. (1993). Spatiotemporal firing patterns in the frontal cortex of behaving monkeys. Journal of Neurophysiology, 70(4), 1629-1638.

Abeles, M., \& Gat, I. (2001). Detecting precise firing sequences in experimental data. Journal of Neuroscience Methods, 107 (1-2), 141-154.

Abeles, M., Hayon, G., \& Lehmann, D. (2004). Modeling compositionality by dynamic binding of synfire chains. Journal of Computational Neuroscience, 17(2), 179-201.

Amari, S.-i. (1977). Dynamics of pattern formation in lateralinhibition type neural fields. Biological Cybernetics, 27, 7787.

Arnoldi, H. M., \& Brauer, W. (1996). Synchronization without oscillatory neurons. Biological Cybernetics, 74(3), 209-223.

Baras, D., \& Meir, R. (2007). Reinforcement learning, spiketime-dependent plasticity, and the BCM rule. Neural Computation, 19, 2245-2279.

Ben-Shaul, Y., Drori, R., Asher, I., Stark, E., Nadasdy, Z., \& Abeles, M. (2004). Neuronal activity in motor cortical areas reflects the sequential context of movement. Journal of Neurophysiology, 91(4), 1748-1762 (comparative study).

Berger, D., Borgelt, C., Louis, S., Morrison, A., \& Grün, S. (2010). Efficient identification of assembly neurons within massively parallel spike trains. Computational Intelligence and Neuroscience, 2010, 439648.

Bienenstock, E. (1995). A model of neocortex. Network: Computational Neural Systems, 6, 179-224.

Bienenstock, E. (1996). Composition. In A. Aertsen \& V. Braitenberg (Eds.), Brain theory-Biological basis and computational principles (pp. 269-300). Amsterdam: Elsevier.

Bizzi, E., Cheung, V., d'Avella, A., Saltiel, P., \& Tresch, M. (2008). Combining modules for movement. Brain Research Reviews, 57(1), 125-133. Networks in Motion.

Bizzi, E., Mussa-Ivaldi, F., \& Giszter, S. (1991). Computations underlying the execution of movement: A biological perspective. Science, 253(5017), 287-291.
Brunel, N. (2000). Dynamics of sparsely connected networks of excitatory and inhibitory spiking neurons. Journal of Computational Neuroscience, 8(3), 183-208.

Buonomano, D. V. (2005). A learning rule for the emergence of stable dynamics and timing in recurrent networks. Journal of Neurophysiology, 94, 2275-2283.

Burns, B. D., \& Webb, A. C. (1976). The spontaneous activity of neurones in the cat's visual cortex. Proceedings of the Royal Society of London B, 194, 211-223.

Calabi, E., Olver, P. J., Shakiban, C., Tannenbaum, A., \& Haker, S. (1998). Differential and numerically invariant signature curves applied to object recognition. International Journal of Computer Vision, 26(2), 107-135.

Calabi, E., Olver, P. J., \& Tannenbaum, A. (1996). Affine geometry, curve flows, and invariant numerical approximations. Advances in Mathematics, 124(1), 154-196.

Carpenter, A. F., Georgopoulos, A. P., \& Pellizzer, G. (1999). Motor cortical encoding of serial order in a context-recall task. Science, 283(5408), 1752-1757.

Chang, W., \& Jin, D. Z. (2009). Spike propagation in driven chain networks with dominant global inhibition. Physical Review E, $79(5), 051917$.

Diesmann, M., Gewaltig, M.-O., \& Aertsen, A. (1999). Stable propagation of synchronous spiking in cortical neural networks. Nature, 402(6761), 529-533.

Dombeck, D. A., Graziano, M. S., \& Tank, D. W. (2009). Functional clustering of neurons in motor cortex determined by cellular resolution imaging in awake behaving mice. Journal of Neuroscience, 29(44), 13751-13760.

Donoghue, J. P., Sanes, J. N., Hatsopoulos, N. G., \& Gaal, G. (1998). Neural discharge and local field potential oscillations in primate motor cortex during voluntary movements. Journal of Neurophysiology, 79, 159-173.

Doursat, R., \& Bienenstock, E. (2006). The self-organized growth of synfire patterns. In 10th international conference on cognitive and neural systems (ICCNS). Massachusetts: Boston University.

Dutar, P., Vu, H. M., \& Perkel, D. J. (1998). Multiple cell types distinguished by physiological, pharmacological, and anatomic properties in nucleus HVC of the adult zebra finch. Journal of Neurophysiology, 80(4), 1828-1838.

Eckhorn, R., Bauer, R., Jordan, W., Brosch, M., Kruse, W., Munk, M., et al. (1988). Coherent oscillations: A mechanism of feature linking in the visual cortex? Biological Cybernetics, 60, 121-130.

Farries, M. A., \& Fairhall, A. L. (2007). Reinforcement learning with modulated spike timing-dependent synaptic plasticity. Journal of Neurophysiology, 98, 3648-3665.

Fiete, I. R., Senn, W., Wang, C. Z. H., \& Hahnloser, R. H. R. (2010). Spike-time-dependent plasticity and heterosynaptic competition organize networks to produce long scale-free sequences of neural activity. Neuron, 65, 563-576.

Georgopoulos, A., Kalaska, J., Caminiti, R., \& Massey, J.T. (1982). On the relations between the direction of twodimensional arm movements and cell discharge in primate motor cortex. Journal of Neuroscience, 11(2), 15271537.

Georgopoulos, A., Schwartz, A., \& Kettner, R. (1986a). Neuronal population coding of movement direction. Science, 4771(233), 1416-1419.

Georgopoulos, A. P., Kettner, R. E., \& Schwartz, A. B. (1988). Primate motor cortex and free arm movements to visual targets in three-dimensional space. II. Coding of the direction of movement by a neuronal population. Journal of Neuroscience, 8(8), 2928-2937. 
Georgopoulos, A. P., Merchant, H., Naselaris, T., \& Amirikian, B. (2007). Mapping of the preferred direction in the motor cortex. PNAS, 104(26), 11068-11072.

Georgopoulos, A. P., Schwartz, A. B., \& Kettner, R. E. (1986b). Neuronal population coding of movement direction. Science, $233,1416-1419$.

Gewaltig, M.-O., \& Diesmann, M. (2007). NEST (neural simulation tool). Scholarpedia, 2(4), 1430.

Gewaltig, M.-O., Diesmann, M., \& Aertsen, A. (2001). Propagation of cortical synfire activity: Survival probability in single trials and stability in the mean. Neural Networks, (14), 657673.

Ghosh, S., Putrino, D., Burro, B., \& Ring, A. (2009). Patterns of spatio-temporal correlations in the neural activity of the cat motor cortex during trained forelimb movements. Somatosensory and Motor Research, 26(2-3), 31-49.

Glaze, C., \& Troyer, T. (2008). Temporal variability in a synfire chain model of birdsong. BMC Neuroscience, 9(Suppl 1), 28.

Gray, C. M., \& Singer, W. (1989). Stimulus-specific neuronal oscillations in orientation columns of cat visual cortex. Proceedings of the National Academy of Sciences of the United States of America, 86, 1698-1702.

Hahnloser, R. H., Kozhevnikov, A. A., \& Fee, M. S. (2002). An ultra-sparse code underlies the generation of neural sequences in a songbird. Nature, 419(6902), 65-70.

Hansel, D., Mato, G., Meunier, C., \& Neltner, L. (1998). On numerical simulations of integrate-and-fire neural networks. Neural Computation, 10(2), 467-483.

Hanuschkin, A., Diesmann, M., \& Morrison, A. (2010a). Functional compositionality realized in biological realistic spiking neural networks by synfire chain competition. In Proceedings of the 40th Annual Meeting of the Society for Neuroscience.

Hanuschkin, A., Diesmann, M., \& Morrison, A. (2010b). A reafferent model of song syntax generation in the Bengalese finch. BMC Neuroscience, 11(Suppl 1), 33.

Hanuschkin, A., Herrmann, J. M., Morrison, A., \& Diesmann, M. (2009a). A model of free monkey scribbling based on the propagation of cell assembly activity. BMC Neuroscience, 10(Suppl 1), 300.

Hanuschkin, A., Herrmann, J. M., Morrison, A., \& Diesmann, M. (2009b). Modeling free monkey scribbling by the propagation of synchronous activity. In Proceedings of the 8th Göttingen Meeting of the German Neuroscience Society.

Hanuschkin, A., Kunkel, S., Helias, M., Morrison, A., \& Diesmann, M. (2010). A general and efficient method for incorporating precise spike times in globally timedriven simulations. Frontiers in Neuroinformatics (4)113. doi:10.3389/fninf.2010.00113.

Hart, C. B., \& Giszter, S. F. (2010). A neural basis for motor primitives in the spinal cord. Journal of Neuroscience, 30(4), 1322-1336.

Hayon, G., Abeles, M., \& Lehmann, D. (2005). A model for representing the dynamics of a system of synfire chains. Journal of Computational Neuroscience, 18, 41-53.

Ha $\beta$, J., Blaschke, S., Rammsayer, T., \& Herrmann, J. M. (2008). A neurocomputational model for optimal temporal processing. Journal of Computational Neuroscience, 25(3), 449-464.

Herrmann, M., Hertz, J. A., \& Prügel-Bennett, A. (1995). Analysis of synfire chains. Network, 6, 403-414.

Ikegaya, Y., Aaron, G., Cossart, R., Aronov, D., Lampl, I., Ferster, D., et al. (2004). Synfire chains and cortical songs: Temporal modules of cortical activity. Science, 5670(304), $559-564$
Izhikevich, E. M. (2007). Solving the distal reward problem through linkage of STDP and dopamine signaling. Cerebral Cortex, 17(10), 2443-2452.

Izhikevich, E. M., Gally, J. A., \& Edelman, G. M. (2004). Spiketiming dynamics of neuronal groups. Cerebral Cortex, 14, 933-944.

Jin, D. Z. (2002). Fast convergence of spike sequences to periodic patterns in recurrent networks. Physical Review Letters, 89(20), 208102.

Jin, D. Z. (2009). Generating variable birdsong syllable sequences with branching chain networks in avian premotor nucleus HVC. Physical Review E, 80(5), 051902.

Jin, D. Z., Ramazanoglu, F. M., \& Seung, H. S. (2007). Intrinsic bursting enhances the robustness of a neural network model of sequence generation by avian brain area HVC. Journal of Computational Neuroscience, 23(3), 283-299.

Jun, J. K., \& Jin, D. Z. (2007). Development of neural circuitry for precise temporal sequences through spontaneous activity, axon remodeling, and synaptic plasticity. PLOS ONE, 2(8), e723.

Kozhevnikov, A., \& Fee, M. S. (2007). Singing-related activity of identified HVC neurons in the zebra finch. Journal of Neurophysiology, 97, 4271-4283.

Kumar, A., Rotter, S., \& Aertsen, A. (2010). Spiking activity propagation in neuronal networks: Reconciling different perspectives on neural coding. Nature Reviews Neuroscience, $11,615-627$.

Kunkel, S., Diesmann, M., \& Morrison, A. (2010). Limits to the development of feed-forward structures in large recurrent neuronal networks (submitted).

Lacquaniti, F., Terzuolo, C., \& Viviani, P. (1983). The law relating the kinematic and figural aspects of drawing movements. Acta Psychologica (Amst), 54(1-3), 115-130.

Lapicque, L. (1907). Recherches quantitatives sur l'excitation electrique des nerfs traitee comme une polarization. Journal de Physiologie et de Pathologie Générale, 9, 620-635.

Legenstein, R., Pecevski, D., \& Maass, W. (2008). A learning theory for reward-modulated spike-timing-dependent plasticity with application to biofeedback. PLoS Computational Biology, 4(10), e1000180.

Li, M., \& Greenside, H. (2006). Stable propagation of a burst through a one-dimensional homogeneous excitatory chain model of songbird nucleus HVC. Physical Review E, 74(1), 011918.

Lindén, H., Pettersen, K. H., Tetzlaff, T., Potjans, T. C., Denker, M., Diesmann, M., et al. (2009a). Estimating the spatial range of local field potentials in a cortical population model. BMC Neuroscience, 10(Suppl I), 224.

Lindén, H., Potjans, T. C., Einevoll, G. T., Grün, S., \& Diesmann, M. (2009b). Modeling the local field potential by a large-scale layered cortical network model. In Frontiers in Neuroinformatics. Conference abstract: 2nd INCF congress of Neuroinformatics. doi:10.3389/conf.neuro.11.2009. 08.046.

Mazzoni, A., Panzeri, S., Logothetis, N. K., \& Brunel, N. (2008). Encoding of naturalistic stimuli by local field potential spectra in networks of excitatory and inhibitory neurons. PLoS Computational Biology, 4(12), e1000239.

Mooney, R., \& Prather, J. F. (2005). The HVC microcircuit: The synaptic basis for interactions between song motor and vocal plasticity pathways. Journal of Neuroscience, 25(8), 19521964.

Morrison, A., Aertsen, A., \& Diesmann, M. (2007a). Spiketiming dependent plasticity in balanced random networks. Neural Computation, 19, 1437-1467. 
Morrison, A., Straube, S., Plesser, H. E., \& Diesmann, M. (2007b). Exact subthreshold integration with continuous spike times in discrete time neural network simulations. Neural Computation, 19(1), 47-79.

Murthy, V. N., \& Fetz, E. (1996). Oscillatory activity in sensorimotor cortex of awake monkeys: Synchronization of local field potentials and relation to behavior. Journal of Neurophysiology, 76, 3949-3967.

Mussa-Ivaldi, F. A., \& Bizzi, E. (2000). Motor learning through the combination of primitives. Philosophical Transactions of the Royal Society London, Series B, 355(1404), 17551769.

Mussa-Ivaldi, F. A., Giszter, S., \& Bizzi, E. (1994). Linear combinations of primitives in vertebrate motor control. Proceedings of the National Academy of Sciences of the United States of America, 91, 7534-7538.

Nordlie, E., Gewaltig, M.-O., \& Plesser, H. E. (2009). Towards reproducible descriptions of neuronal network models. PLoS Computational Biology, 5(8), e1000456.

O'Leary, J. G., \& Hatsopoulos, N. G. (2006). Early visuomotor representations revealed from evoked local field potentials in motor and premotor cortical areas. Journal of Neurophysiology, 96(3), 1492-1506.

Plesser, H. E., \& Diesmann, M. (2009). Simplicity and efficiency of integrate-and-fire neuron models. Neural Computation, 21, 353-359.

Polyakov, F., Drori, R., Ben-Shaul, Y., Abeles, M., \& Flash, T. (2009a). A compact representation of drawing movements with sequences of parabolic primitives. PLoS Computational Biology, 5(7), e1000427.

Polyakov, F., Stark, E., Drori, R., Abeles, M., \& Flash, T. (2009b). Parabolic movement primitives and cortical states: Merging optimality with geometric invariance. Biological Cybernetics, 100(2), 159-184.

Ponce-Alvarez, A., Kilavik, B. E., \& Riehle, A. (2010). Comparison of local measures of spike time irregularity and relating variability to firing rate in motor cortical neurons. Journal of Computational Neuroscience, 29(1-2), 351-365.

Potjans, W., Morrison, A., \& Diesmann, M. (2009). A spiking neural network model of an actor-critic learning agent. Neural Computation, 21, 301-339.

Potjans, W., Diesmann, M, \& Morrison, A. (2010). An imperfect dopaminergic signal can drive temporal-difference learning (submitted).

Prut, Y., Vaadia, E., Bergman, H., Haalman, I., Hamutal, S., \& Abeles, M. (1998). Spatiotemporal structure of cortical activity: Properties and behavioral relevance. Journal of Neurophysiology, 79(6), 2857-2874.

Pulvermüller, F., \& Shtyrov, Y. (2009). Spatiotemporal signatures of large-scale synfire chains for speech processing as revealed by MEG. Cerebral Cortex, 19, 79-88.

Putrino, D., Brown, E. N., Mastaglia, F. L., \& Ghosh, S. (2010). Differential involvement of excitatory and inhibitory neurons of cat motor cortex in coincident spike activity related to behavioral context. Journal of Neuroscience, 30(23), 80488056.

Rickert, J., Cardoso de Oliveira, S., Vaadia, E., Aertsen, A., Rotter, S., \& Mehring, C. (2005). Encoding of movement direction in different frequency ranges of motor cortical local field potentials. Journal of Neuroscience, 25(39), 88158824.

Riehle, A., Grammont, F., Diesmann, M., \& Grün, S. (2000). Dynamical changes and temporal precision of synchronized spiking activity in monkey motor cortex during movement preparation. Journal of Physiology (Paris), 94(5-6), 569-582. Erratum in "Journal of Physiology (Paris)", 95(1-6), 499 (2001).

Riehle, A., Grün, S., Diesmann, M., \& Aertsen, A. (1997). Spike synchronization and rate modulation differentially involved in motor cortical function. Science, 278(5345), 19501953.

Rioult-Pedotti, M. S., Friedman, D., Hess, G., \& Donoghue, J. P. (1998). Strengthening of horizontal cortical connections following skill learning. Nature Neuroscience, 1, 230234.

Rotter, S., \& Diesmann, M. (1999). Exact digital simulation of time-invariant linear systems with applications to neuronal modeling. Biological Cybernetics, 81(5-6), 381-402.

Roux, S., Mackay, W. A., \& Riehle, A. (2006). The premovement component of motor cortical local field potentials reflects the level of expectancy. Behavioural Brain Research, $169(2), 335-351$.

Schrader, S., Diesmann, M., \& Morrison, A. (2010). A compositionality machine realized by a hierarchic architecture of synfire chains (submitted).

Schrader, S., Grün, S., Diesmann, M., \& Gerstein, G. (2008). Detecting synfire chain activity using massively parallel spike train recording. Journal of Neurophysiology, 100, 21652176.

Schwartz, A. B. (1994). Direct cortical representation of drawing. Science, 265(5171), 540-542.

Sforza, C., Turci, M., Grassi, G., Fragnito, N., Pizzini, G., \& Ferrario, V. (2000). The repeatability of choku-tsuki and oi-tsuki in traditional shotokan karate: A morphological three-dimensional analysis. Perceptual and Motor Skills, 90, 947-960.

Shmiel, T., Drori, R., Shmiel, O., Ben-Shaul, Y., Nadasdy, Z., Shemesh, M., et al. (2005). Neurons of the cerebral cortex exhibit precise interspike timing in correspondence to behavior. PNAS, 102(51), 18655-18657.

Shmiel, T., Drori, R., Shmiel, O., Ben-Shaul, Y., Nadasdy, Z., Shemesh, M., et al. (2006). Temporally precise cortical firing patterns are associated with distinct action segments. Journal of Neurophysiology, 96(5), 2645-2652.

Singer, W., \& Gray, C. (1995). Visual feature integration and the temporal correlation hypothesis. Annuual Review of Neuroscience, 18, 555-586.

Softky, W. R., \& Koch, C. (1993). The highly irregular firing of cortical cells is inconsistent with temporal integration of random EPSPs. Journal of Neuroscience, 13(1), 334350.

Stark, E., Drori, R., \& Abeles, M. (2009). Motor cortical activity related to movement kinematics exhibits local spatial organization. Cortex, 45, 418-431.

Tetzlaff, T., Geisel, T., \& Diesmann, M. (2002). The ground state of cortical feed-forward networks. Neurocomputing, 44-46, 673-678.

Ursino, M., \& Cara, G.-E. L. (2006). Travelling waves and EEG patterns during epileptic seizure: Analysis with an integrateand-fire neural network. Journal of Theoretical Biology, 242(1), 171-187.

van Vreeswijk, C., \& Sompolinsky, H. (1996). Chaos in neuronal networks with balanced excitatory and inhibitory activity. Science, 274, 1724-1726.

Viviani, P., \& Flash, T. (1995). Minimum-jerk, two-thirds power law, and isochrony: Converging approaches to movement planning. Journal of Experimental Psychology: Human Perception and Performance, 21(1), 32-53. 
Wennekers, T., \& Palm, G. (1996). Controlling the speed of synfire chains. In C. von der Malsburg, W. von Seelen, J. C. Vorbrüggen, \& B. Sendhoff (Eds.), Artificial neural networks-ICANN 96 (pp. 451-456). Berlin: Springer-Verlag.

Xu, T., Yu, X., Perlik, A. J., Tobin, W. F., Zweig, J. A., Tennant, K., et al. (2009). Rapid formation and selective stabilization of synapses for enduring motor memories. Nature, 462, 915919.

Yamashita, Y., \& Tani, J. (2008). Emergence of functional hierarchy in a multiple timescale neural network model: A humanoid robot experiment. PLoS Computational Biology, 4(11), e1000220. 